\title{
Algorithmic Design Issues in Adaptive Differential Evolution Schemes: Review and Taxonomy
}

\author{
Rawaa Dawoud AL-Dabbagh Ferrante Neri Norisma Idris Mohd Sapiyan Baba
}

\begin{abstract}
The performance of most metaheuristic algorithms depends on parameters whose settings essentially serve as a key function in determining the quality of the solution and the efficiency of the search. A trend that has emerged recently is to make the algorithm parameters automatically adapt to different problems during optimization, thereby liberating the user from the tedious and time-consuming task of manual setting. These fine-tuning techniques continue to be the object of ongoing research. Differential evolution (DE) is a simple yet powerful population-based metaheuristic. It has demonstrated good convergence, and its principles are easy to understand. DE is very sensitive to its parameter settings and mutation strategy; thus, this study aims to investigate these settings with the diverse versions of adaptive DE algorithms. This study has two main objectives: (1) to present an extension for the original taxonomy of evolutionary algorithms (EAs) parameter settings that has been overlooked by prior research and therefore minimize any confusion that might arise from the former taxonomy and (2) to investigate the various algorithmic design schemes that have been used in the different variants of adaptive DE and convey them in a new classification style. In other words, this study describes in depth the structural analysis and working principle that underlie the promising and recent work in this field, to analyze their advantages and disadvantages and to gain future insights that can further improve these algorithms. Finally, the interpretation of the literature and the comparative analysis of the results offer several guidelines for designing and implementing adaptive DE algorithms. The proposed design framework provides readers with the main steps required to integrate any proposed meta-algorithm into parameter and/or strategy adaptation schemes.
\end{abstract}

Keywords Metaheuristic algorithms; Optimization algorithms; Evolutionary Algorithms; Genetic Algorithm; Parameter Control; Differential Evolution; Adaptive Differential Evolution; Review

Rawaa Dawoud AL-Dabbagh, Corresponding Author

Department of Computer Science,

University of Baghdad, Baghdad, Iraq

E-mail: rawaa.dabbagh@ieee.org

\section{Ferrante Ner}

Centre for Computational Intelligence,

The Gateway, De Montfort University, UK

E-mail: fneri@dmu.ac.uk

\section{Norisma Idris}

Department of Artificial Intelligence,

University of Malaya, Kuala Lumpur, Malaysia

Email: norisma@um.edu.my

\section{Mohd Sapiyan Baba}

Computer Science Department

Gulf University for Science and Technology, Kuwait

Email: sapiyan.m@gust.edu.kw 


\section{Introduction}

Real-world optimization problems are found in a wide variety of applications, such as machine learning, system design and nearly all areas (i.e. disciplines) of science and engineering. The extensive research interest on providing the best solutions to these problems is ongoing. The main challenge such research confronts is often related to uncertainties and/or noise that can lead to theoretical optima, which are by no means optimal or practical in real life (Arnold and Beyer 2002; Jin and Branke 2005). Efficient algorithms are known and used to solve typical optimization problems, whereas heuristic methods are used to solve difficult optimization problems (Junior et al. 2012a; Junior et al. 2012b). However, the increase in the complexity of the problems, in conjunction with the need to decrease the time consumed for thorough problem analysis and tailored algorithm design, implies a demand for robust algorithms with satisfactory performance. These algorithms should not merely adhere to specific problems but are applicable to a wide range of problems and yield good solutions (although unnecessarily optimal) (Eiben and Smith 2003; Gendreau and Potvin 2010; Salvatore et al. 2010). Under this class of optimization algorithms are metaheuristic algorithms, such as evolutionary algorithms (EAs) and swarm intelligence algorithms (SIA), which are used to find the parameters (or structures) that maximize or minimize user-defined objective functions. The development of these algorithms continues to be heated and remains an ongoing research task (Boussaï et al. 2013; Zhang et al. 2017). Consequently, algorithmic designers or developers must consider another challenge in addition to problem specifics; it is the well design of the intrinsic part of the meta-algorithm itself (Yang et al. 2013). This includes certain algorithm specifics or capabilities, such as maintaining the diversity and mixing among the solutions. Well-designed algorithms must have such capabilities retained for balancing global exploration and intensive local exploitation given that these two antagonisms constitute the efficient search ability (Fister et al. 2013).

The dialects of all metaheuristics are generally based on the same generic framework whose details need to be specified to obtain a particular algorithm. These details are customarily called algorithm parameters, such as probability of mutation, probability of crossover, tournament size of selection and size of population. Many studies on EAs examine the implementation of adaptive EAs (Cotta et al. 2008; Lobo et al. 2007). This type of EAs, if well designed, can enhance the robustness and convergence performance of the algorithm by dynamically updating the EA parameters for different objective function landscapes during evolution (Karafotias et al. 2015). This has led to the on-the-fly alteration for such parameters during evolution by accounting for the actual search progress to achieve optimal convergence and liberate a user from the tedious trials of tuning the parameters. As discussed in (Eiben et al. 1999) and (Eiben and Smith 2003), the main idea is to no longer choose the parameters in a semi-arbitrary fashion ${ }^{1}$ but to allow the parameters to adapt themselves to the problem.

More than a decade ago, differential evolution (DE) algorithm emerged as a special and competitive form of metaheuristics (Das et al. 2016). Owing to its distinct features, DE algorithm can be viewed from two different perspectives. It can be classified as EA because of its recombination and selection operators, in addition to the names and descriptions of its control parameters. At the same time, DE algorithm can also be regarded as SIA because of its structure that, over a number of generations, a group of initiated particles tend to converge their values closer to the particle whose value is closest to the target at any given moment (Panigrahi et al. 2011; Weber et al. 2010). Notwithstanding of its classification, DE algorithm and its numerous variants have developed rapidly as simple and robust algorithms. Practitioners from different disciplines of science and engineering have applied DE algorithms to address various optimization problems in their own fields, regardless of whether these problems are continuous, combinatorial or mixed variable (Abderazek et al. 2017; Al-Dabbagh et al. 2015a). DE has produced superior results across widely used benchmark functions (Elsayed et al. 2014;

\footnotetext{
${ }^{1}$ The choices were often based on experience.
} 
Wang et al. 2012) and real-world applications (Goudos et al. 2017; Zhang et al. 2010). Table 1 covers excerpts of the most prominent milestones and epochs in the DE history starting from the time it was proposed by Storn and Price in 1995 (Storn and Price 1995a; Storn and Price 1997) until present. The events in Table 1 are presented chronologically. This arrangement can be observed from the years that correspond to each subject (line). However, certain topics require citations from past to recent years. Consequently, two markers have been used in the Juncture column. The first marker is a small circle that refers to a specific work or publication in the $\mathrm{DE}$ literature. The second marker is a line that refers to an unspecified number of publications in the DE literature that are related to one topic starting from the year during which the first work regarding that topic was published. For a subject with a line marker, related and recent references are cited as examples.

Claims and counterclaims have already been proposed, especially by engineers, regarding the rules in choosing the appropriate control values of standard DE parameters with which to solve practical problems (Feoktistov 2006; Zou et al. 2011). However, the performance of DE algorithm depends heavily on the selected mutation strategy and its associated control parameters. The sensitivity of the DE algorithm to its mutation strategy and its corresponding control parameters can significantly deteriorate its performance if the strategy is improperly selected (Al-Dabbagh et al. 2015b; Wang et al. 2013). Choosing a suitable DE strategy and setting its control parameters is difficult and requires much user experience. In the past few years, many researchers have attempted to make the algorithm into a general and fast optimization method for any kind of optimization problem by tuning its various constitutes, such as initialization, mutation, diversity enhancement, and crossover of DE (Awad et al. 2017a; Das et al. 2014a). Multiple attempts have also been conducted to automatically adjust the algorithm's parameters for single or multiple problems. The development of (self-)adaptive DE algorithms has resulted in faster and more reliable convergence performance in many benchmark problems than classical DE algorithms with manual parameter settings (Brest et al. 2007; Zhang and Sanderson 2009b; Zhu et al. 2013).

The present study aims to provide insights for fresh and experienced practitioners alike who are interested in the field of parameter settings in metaheuristics. This study is designed from the ground up to support the issue of controlling the values of various parameters of an evolutionary algorithm in general and of DE algorithm in particular. The main objective of this study is to present a comprehensive procedural analysis (in SECTION 5) that is conducted to investigate the various adaptive schemes utilized to automatically control the DE parameters and/or its mutation strategies. For this purpose, two taxonomies are proposed in this study. The first taxonomy (in SECTION 3-Fig 2) is proposed to eliminate any ambiguity related to classify any adaptive EA. The new classification comprises three levels of categories instead of two regarding the parameter control type (deterministic, adaptive, self-adaptive) and the evidence (absolute, relative) used for determining the change of the parameter. The second taxonomy (in SECTION 5-Fig 3) is a new taxonomy proposed to classify the adaptive DE algorithms into two categories (DE with adaptive parameters and DE with adaptive parameters and mutation strategies). This study comprehensively describes the structural analysis and working principle that underlie the promising work of these algorithms. The study also discusses the advantages and disadvantages of these algorithms and suggests future insights that can further improve their algorithmic design. Eventually, protocols for future adaptive DE implementations are also offered (in SECTIONS 6-7).

The rest of this paper is organized as follows. SECTION 2 provides an overview of the published survey work on adaptive DE algorithms. SECTION 3 presents an extended taxonomy of EA parameter settings. SECTION 4 discusses the standard procedural design of DE algorithm. SECTION 5 applies the two proposed taxonomies (in Fig 2 and Fig 5) to multiple adaptive DE algorithms specifically as an example to convey the main purpose of these taxonomies. A procedural analysis is then established on these algorithms to elucidate the conceptual similarities and differences among them and the pros and cons of each adaptive scheme. SECTION 6 presents a general framework that lists the steps that should be considered to create a meta-algorithm with parameter control. SECTION 7 concludes the paper and summarizes the objectives addressed. Suggestions and future work developments are also offered in this section. 


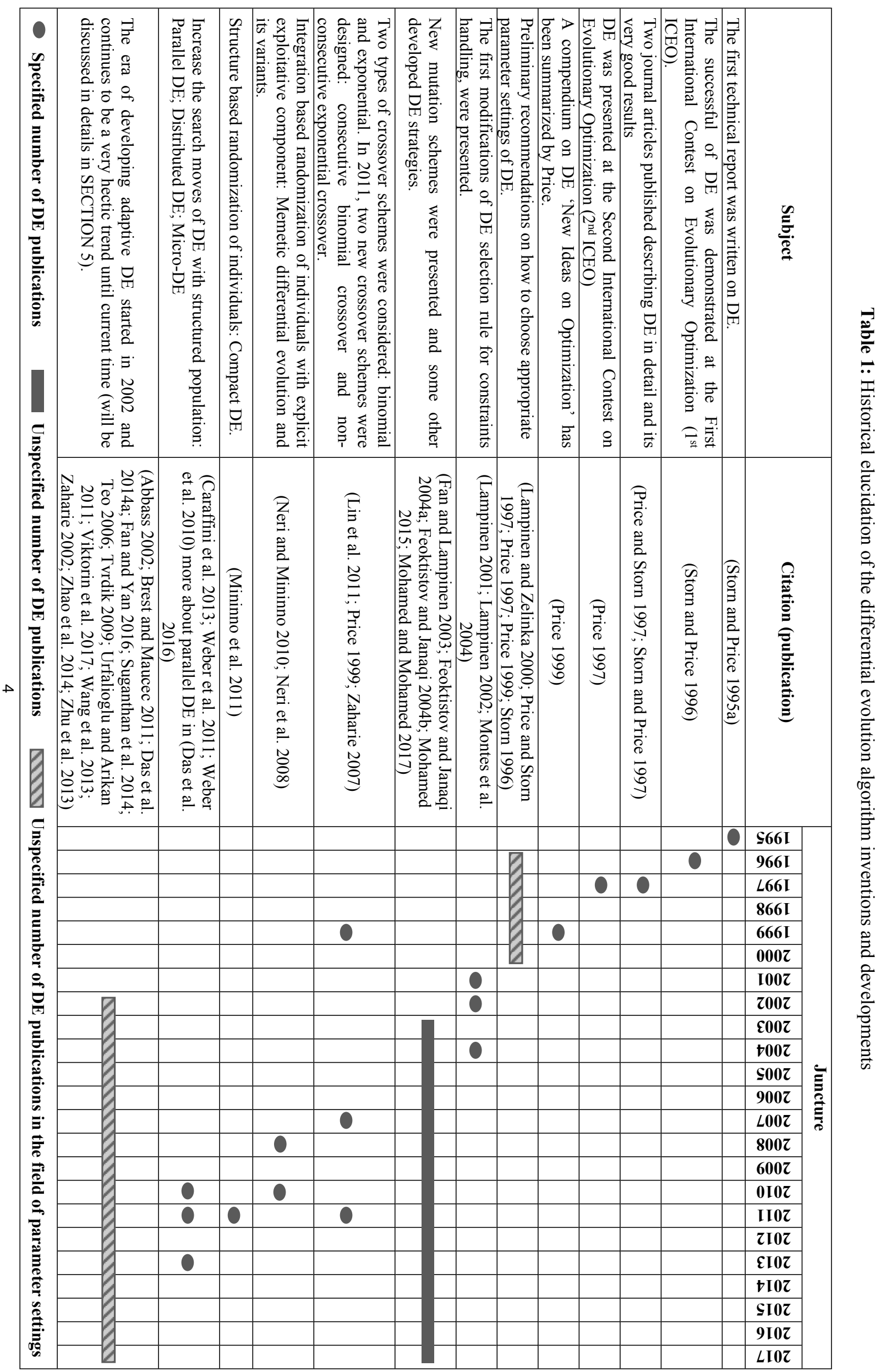




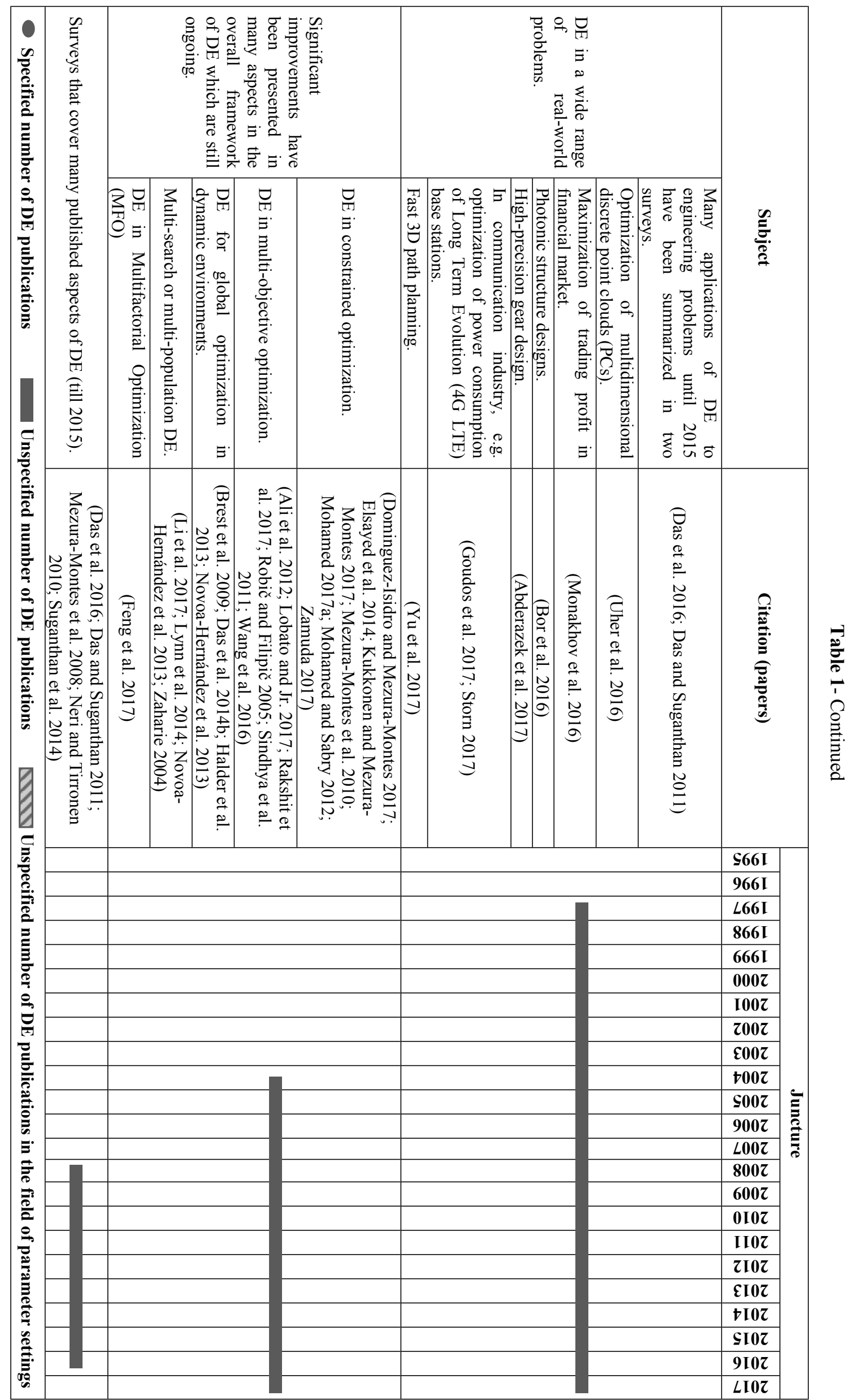




\section{Related state-of-the-art work: in what way is this survey different to the others?}

Since numerous DE variants have been proposed in the literature, many surveys on DE have been published. Several survey papers have fully captured the performance aspects of adaptive and self-adaptive DE. Das and Suganthan (2011) published a comprehensive survey that addressed nearly all scenarios where DE is applied, such as DE and constrained optimization functions, important DE schemes for single-objective functions, DE in complex environments, theoretical analysis development of DE and most contemporary engineering applications of DE. The review of Das and Suganthan (2011) also briefly introduced the control of DE parameters and presented the most prominent and recent DE variants in the field. The survey of Neri and Tirronen (2010) presented DE algorithms and their most recent advances in a classification format. The reviewed algorithms were categorized into two classes. The first class is based on integrating DE with an additional component, such as local search methods. The second class is based on modifying the DE structure, such as algorithms with adaptive schemes. The study also conducted detailed experiments based on a wide set of benchmark problems to test the overall performance of these algorithmic classes.

Chiang et al. (2013) published a new taxonomy for DE parameter control mechanisms based on the type of parameter values (discrete and continuous), number of parameter values (multiple, individual and variable) and the information used to adjust the parameter values (random, population, parent and individual).

Suganthan et al. (2014) published a review paper on adaptive DE variants. In their review, the authors introduced a simple classification of adaptive $\mathrm{DE}$ algorithms according to the type of optimization problem that they are dealing with: adaptive $\mathrm{DE}$ for a single optimization problem, adaptive $\mathrm{DE}$ for other problem scenarios and adaptive population topology in DE. Several adaptive DE algorithms, such as DE with a self-adaptive trial vector generator (Brest et al. 2006) and DE with an adaptive trial vector generator and parameter control (Qin et al. 2009) have been listed under the three proposed classes. Although several adaptive DE algorithms with applications in multiple problem scenarios were included in this literature survey, no general adaptive classification that covered the different aspects of published adaptive DE was proposed. Moreover, no thorough procedural analysis with the pros and cons of these algorithms was provided. Lastly, this review is obsolete.

Das et al. (2016) published an updated version of Das and Suganthan's 2011 survey paper. In their work, a complete section was devoted to cover recent adaptive DE algorithms in a classification format, such as DE with adaptive scalar factor $(F)$ and crossover rate $(C R)$ parameters only, DE with both adaptive strategy and control parameters $(F$ and $C R)$ and DE with adaptive population size $(N p)$. However, we wish to raise some points about this section of the survey.

- Algorithms included under this classification are dated between 2011 and 2015. New versions of LSHADE, such as LSHADE-cnEpSin (Awad et al. 2017b), have been published recently. We have referred to these versions in our study and other new versions of adaptive DEs have also been cited and discussed.

- We have found algorithms with special adaptive properties that have been included in classes with different adaptive merits. For example, Sarker et al. (2014) and Tanabe and Fukunaga (2014) are included in the class where $F$ and $C R$ are the only adaptive parameters; however, these algorithms also update the value of $N p$. Similarly, Zamuda et al. (2013) is included in the class where $F$ and $C R$ are the only adaptive parameters; however, this work also adapts the $N p$ value and the trial vector generation schemes. Finally, Brest and Maucec (2011), Zamuda and Brest (2012) and Zamuda et al. (2013) are included in the class of adaptive DE with $N p$; however, these algorithms also exhibit the adaptation property of multiple mutation strategies, which can be overlooked by readers.

- The discussion about the impact of the type of selected mutation strategy and parameter adaptation scheme is nearly missing. The analysis about the pros and cons of each algorithm (such as in SECTION 
5) and future work that can be conducted to extend the referenced studies (such as in SECTION 7) are also missing.

- We have thoroughly discussed a critical issue regarding adaptive algorithms in our paper, which has been overlooked by many survey studies on this topic. The issue being referred to is the classification of parameter control (and its extended version) in any metaheuristic approach (SECTION 3). This classification is proposed to eliminate any ambiguity related to the classification and understanding of the adaptive characteristics of any adaptive metaheuristic and to complete any survey related to adaptive algorithms. For example, an adaptive probability-based scheme was used to adapt mutation strategies in (Bujok et al. 2014) according to the 2016 survey of Das et al. By contrast, a deterministic parameter control scheme was used to select the values of $F$ and $C R$ for each strategy during the run, which makes this algorithm different from others. These details concerning adaptive algorithms must be highlighted.

Moreover, a survey paper typically attracts new researchers in the field. Thus, sufficient background about the topic it addresses should be provided.

Finally, the main objectives of these review papers are to report, classify and categorize DE versions presented in the literature. Unlike them, the current article focuses on adaptation with the purpose to perform the algorithmic design of $\mathrm{DE}$ algorithms. The interpretation of the literature and the comparative analysis of the results offer several guidelines on how to design and implement adaptive DE algorithms (SECTION 6). The proposed designing framework provides readers with the main steps required to integrate any proposed metaalgorithm into parameter and/or strategy adaptation schemes. Moreover, we design a state-of-the-art DE schematic flow by customizing a distinct alphabetical index for each contribution aspect considering the strong and multifaceted contribution trends of the DE algorithm and our tendency towards simplicity, as depicted in Fig 1. As shown in the figure, some or even all areas of a DE algorithm can overlap in a common work. We eventually found that surveys that deal with the control of DE parameters (Fig 1-b.2) are rare or obsolete (Chiang et al. 2013; Das et al. 2016). This research gap has encouraged us to conduct the current comprehensive survey to cover certain aspects related to DE parameter control.

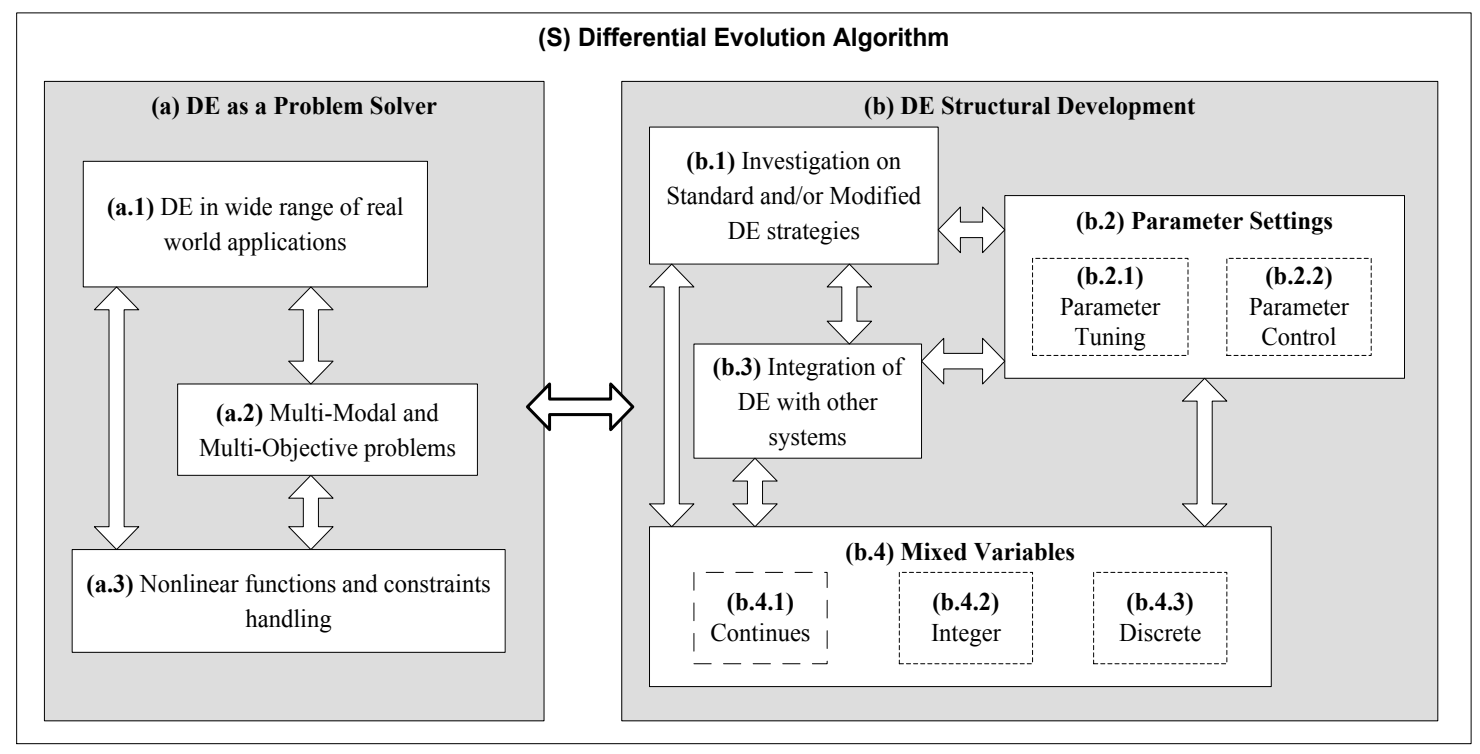

Fig 1: Combination of differential evolution state-of-the-art flow diagram 


\section{Metaheuristics parameter settings: Extended taxonomy}

The critical decision in implementing any meta-algorithm is on how to set the values for various parameters of that algorithm. These values greatly affect the performance of the evolution. Parameter settings are commonly composed of crossover rate, mutation step, population size, selection pressure, and penalty coefficient. It is an important and promising aspect of metaheuristics. As an instance, the efficiency of an EA greatly depends on the setting of these parameters, that is, by parameter tuning or parameter control (Al-Dabbagh et al. 2015b; Karafotias et al. 2015). Parameter tuning is also called off-line setting and involves using several "standard" parameter setting values in advance and keeping these settings fixed during the run, whereas parameter control is also called on-line setting and involves using another class of approaches where parameters are subject to change or evolve as problem parameters are optimized. Scientists and practitioners typically tune EA parameters manually and are guided only by their experience and some rules of thumb. Parameter tuning often requires tedious and time-consuming human involvement. Moreover, the process of any EA is essentially adaptive and dynamic process; thus, using fixed parameters with constant values opposes this essence. Intuitively, the values of these parameters might be optimal at different stages of the evolution; any effort spent toward this direction is indeed lost a priori (Angeline 1995; Brest et al. 2007; Cotta et al. 2008; Eiben et al. 1999; Eiben and Smith 2003). The downsides or limitations of parameter tuning are as follows (Lobo et al. 2007):

- Parameter values tuned for a single problem may lead to a large difference in performance if these parameters were set to different values.

- A parameter tuned for one test problem and produced superior results may not be as effective in other problems.

- EA parameters are intrinsically dependent; thus, tuning them independently is inconvenient.

An alternative form is parameter control, which refers to when an automated setting is applied on EA parameter values. Globally, the automation of parameter settings encompasses three main categories (Cotta et al. 2008; Eiben and Smith 2003; Lobo et al. 2007):

- Deterministic parameter control - automation occurs when a deterministic rule is triggered to modify the value of a strategy parameter in a fixed, predetermined manner without using any feedback from the search.

- Adaptive parameter control - automation occurs during the evolution when the strategy parameter direction and/or magnitude are adjusted according to a pre-designed rule. Basically, automation incorporates information gleaned from the feedback based on algorithm performance, such as the quality of the individual fitness value, without being part of the evolution, where the new control parameter value may or may not persists or propagates throughout the next iterations.

- Self-adaptive parameter control - automation occurs when the strategy parameters undergo genetic encoding and when the alteration is subject to evolution and pressure (i.e., mutation and crossover); better parameter values tend to produce better individuals (i.e., solutions) with the highest chance to survive and propagate for more off-springs.

Another important criterion that should be considered when discussing parameter control techniques is the evidence of change in parameter value, which can be observed from the performance of operators, the diversity of the population, and fitness values. Evidence can be absolute or relative. Absolute evidence is when a rule is applied to alter a strategy parameter value on the basis of a predefined event feedback, such as updating the probability of mutation rate in accordance with a fuzzy rule set, population diversity drops at some given value, and even time elapses, rather than being relative to the performance of other values. By contrast, relative 
evidence is when the strategy parameter value is altered according to the fitness of the offspring produced and the better is rewarded; this change is specified relative, not deterministically, to one value present at any time. Therefore, deterministic parameter control is impossible with relative evidence and thus for self-adaptive parameter control with absolute evidence (Angeline 1995; Cotta et al. 2008; Eiben et al. 1999; Eiben and Smith 2003).

The aforementioned terminologies of the parameter setting of EAs have led to the taxonomy illustrated in Fig. 2. The new taxonomy is an extension of a former one suggested in (Eiben and Smith 2003) which caused some confusion among a number of researchers working in this field, particularly in distinguishing deterministic and absolute adaptive rule, as well as relative adaptive rule and self-adaptive rule.

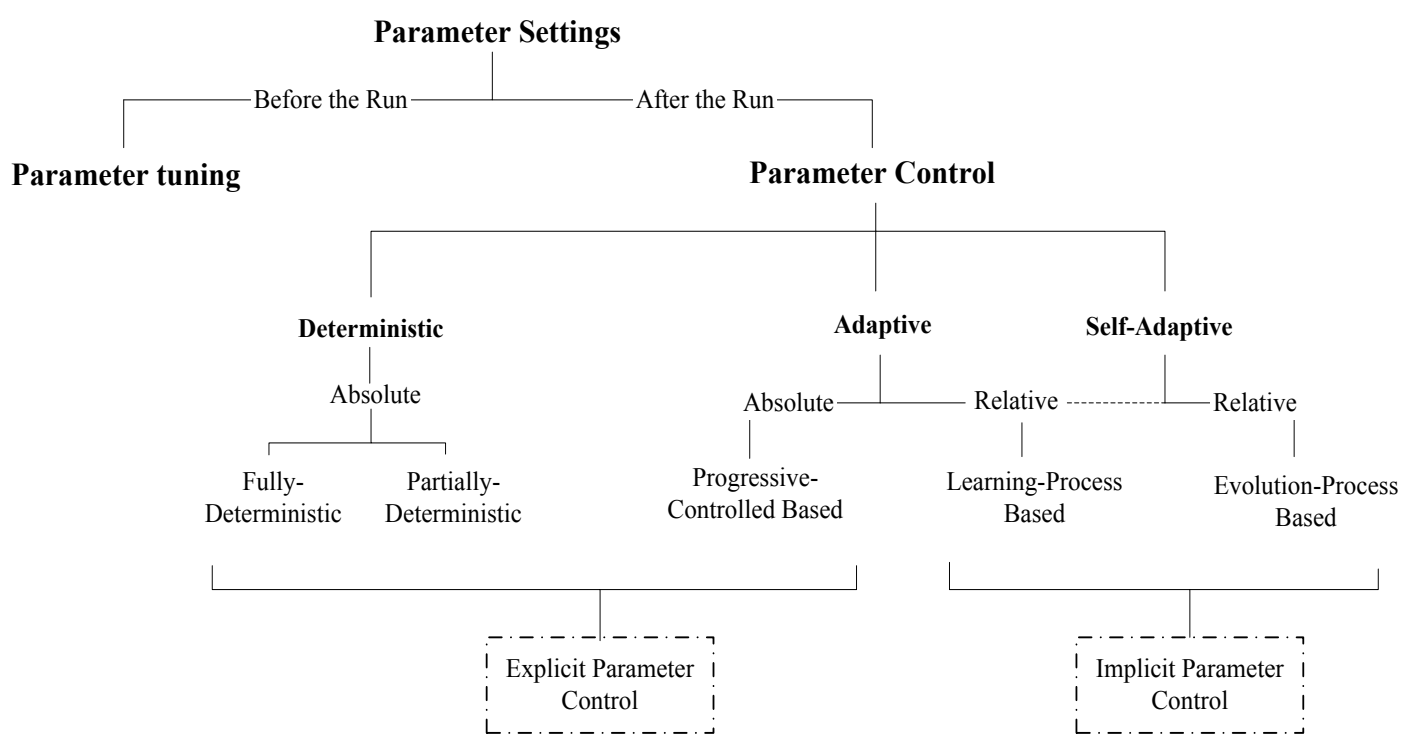

Fig 2: Extended taxonomy of parameters settings in EAs

Accordingly, we investigated the subject of parameter control and found new subcategories that can be added to the main one to address the ambiguity in classification. The definitions of these subcategories are as follows:

- Fully-Deterministic Scheme and Partially-Deterministic Scheme, these two subcategories fall under Deterministic Parameter Control category. Their main feature is not receiving any feedback from the search during the evolution. Technically, a fully-predetermined rule is when a user makes a complete counter-intuition on how to steer the control parameter to the desired direction and/or magnitude; for instance, a rule is triggered on the basis of a certain number of generations that elapsed. By contrast, a partially-predetermined rule is when one uses random based scheme to alter, for example, mutation probability after every 100 generations (Fogel et al. 1991).

- Progressive-Controlled Based, this subcategory is applied when some feedback from the search is discriminated as measurements on the basis of user pre-determined rules. When these measurements achieve a threshold, a corresponding adaptive rule is applied to update its relative parameter control. Thus, the progress of updating parameter values, as well as the search, is controlled under inconsiderable intuition. For instance, these measurements may be based on gathering information from previous runs through data mining-based fuzzy-knowledge control (Liu and Lampinen 2005), theoretical considerations (Smith and Smuda 1995), or practical experience encapsulation (Lis 1996). A 
prominent example of this type of parameter control is the $1 / 5$ success rule of Rechenberg (Rechenberg 1973; Schwefel 1977); this rule is applied at certain periodic intervals deterministically.

- Progressive-Uncontrolled Based (Learning Process-Based), this subcategory is the most prominent in literature. This sub-classification falls between adaptive rule with relative evidence and self-adaptive with evolution-process rule, because both rules are intersected on the basis of updating the control parameter values associated with each individual, at each generation based on their corresponding fitness value; better features of individuals will be propagated to the next populations over time. The only difference is that in progressive-uncontrolled rule feedback is gained from the search to allow the parameter control to gradually adapt by applying a pre-specified strategy, which is most likely analogue to that of crossover and mutation strategies (Hansen and Ostermeier 1996; Qin and Suganthan 2005; Zhang and Sanderson 2009b); most of these strategies are learning schemes that collect experience from previous search. In such methods, the changes performed on the parameter values are fully uncontrolled, because the adaptive rule is associated only with the "fittest" solutions and its corresponding control parameters. This subcategory causes much confusion for some researchers working in this field (Zhang and Sanderson 2009b). For convenience, we use dashed-line connector to make it optional for researchers who desire to stick with the former taxonomy, not to use the latter one, and include the learning style under self-adaptive category, as shown in Fig 2.

Categories that are involved in the search and gradually evolved by either learning or evolution strategy as long as the search is not yet terminated, are considered as implicit parameter control, otherwise, are explicit parameter control.

Ultimately, recent studies have examined the inclusion of algorithms' operators under the new extended classification (i.e. Fig. 2) as well to yield new algorithms with adaptive parameters and operators. These algorithms have shown substantial performance improvements compared with algorithms with adaptive parameters only (Hui and Suganthan 2016; Poláková 2017). Accordingly, self-adaptive algorithms are defined as algorithms with operators and parameters encoded together with their associated solutions and evolved during the run, whereas adaptive algorithms are defined as separate adaptation-based performance algorithms in which parameters and operators that yield good solutions are rewarded and propagated to the next generations (Suganthan 2016), as discussed in detail in SECTION 5.

\section{Differential Evolution: Standard structure}

Differential evolution (DE) is a robust and competitive metaheuristic method for real parameter optimization (Storn and Price 1995b). Its effectiveness in solving a wide variety of optimization problems in science and engineering domains has been proven (Das and Suganthan 2011). This effectiveness is due to its simple concept, which makes it easy to implement. Classical DE features few control parameters (scalar factor $F$, crossover rate $C R$ and population size $N p$ ), although they greatly affect the performance of DE (Teo 2006; Zhang and Sanderson 2009b) which will discussed in the next section. After the initialization stage, DE enters a flow cycle of stages (mutation, crossover and selection) which is repeated in that order for $G_{\max }$ generations.

\subsection{DE vector initialization}

DE, like many EAs, is a population-based optimizer that starts attacking the problem by generating multiple, uniformly and randomly chosen points as initial samples to create population $P$. Usually, each point $x$ of $P$ represents a vector of $D$-dimension in a real parameter space $\mathbb{R}^{\mathrm{D}}$ like $x=\left[x_{1}, x_{2}, \ldots, x_{D}\right]$ and is indexed with a number between 1 and $N p$. In the first generation $(G=0)$, these vectors are initiated according to upper $x_{\min }=$ [ 
$\left.x_{1, \min }, x_{2, \min }, \ldots, x_{D, \min }\right]$ and lower $x_{\max }=\left[x_{1, \max }, x_{2, \max }, \ldots, x_{D, \max }\right]$ bounds of each decision variable (problem parameter) $x_{j}$ of $x$ as

$$
x^{G=0}=x_{j, i} \min +\operatorname{rand}[0,1]_{j, i} \cdot\left(x_{j, \max }-x_{j, \min }\right)
$$

where rand $[0,1]$ is a uniformly distributed random number generated between 0 and 1.

In DE community, the vectors generated at each stage of DE cycle have different names. For example, the vectors or population created at the beginning of each generation are called target vectors/target population and denoted as $x$.

\subsection{DE mutation operation}

In the preceding stage of generating the target vectors $x_{i}(i=1,2, \ldots, N p)$, mutation operation is performed to generate donor vectors/donor population $v_{i}(i=1,2, \ldots, N p)$. The main idea of this operation (see Eq. 2 ) is to add a scaled difference of two target vectors $\left(x_{r 1}\right.$ and $\left.x_{r 2}\right)$ to a third vector $x_{r 3}$ from the current population to yield the mutant vector $v_{i}$. The three indices $(r 1, r 2$ and $r 3)$ are mutually distinct, chosen from the range $[1, N P]$, and do not coincide with the index $i$ of the current vector. This operation is also called differential mutation.

$$
v_{i}^{G}=x_{r 1}^{G}+F \cdot\left(x_{r 2}^{G}-x_{r 3}^{G}\right)
$$

In Eq. 2, $G$ represents the current generation, and $F$ is a control parameter that determines the exploration length $\left(x_{r 2}-x_{r 3}\right)$ governing how far the offspring from point $x_{r 1}$ should be generated. $F \in[0,1+[$ always takes positive value which cannot be greater than 1 (Price et al. 2005). DE has many nomenclatures in the literature derived from its mutation formulas. The DE scheme shown in Eq. 2 is called DE/rand/1. Other DE schemes are expressed below.

$\mathrm{DE} /$ best $/ 1$

$$
v_{i}^{G}=x_{b e s t}^{G}+F \cdot\left(x_{r 1}^{G}-x_{r 2}^{G}\right)
$$

DE/current-to-best/1

$$
\begin{aligned}
& v_{i}^{G}=x_{i}^{G}+F \cdot\left(x_{\text {best }}^{G}-x_{i}^{G}\right)+F \cdot\left(x_{r 1}^{G}-x_{r 2}^{G}\right) \\
& v_{i}^{G}=x_{r 1}^{G}+F \cdot\left(x_{r 2}^{G}-x_{r 3}^{G}\right)+F \cdot\left(x_{r 4}^{G}-x_{r 5}^{G}\right) \\
& v_{i}^{G}=x_{\text {best }}^{G}+F \cdot\left(x_{r 1}^{G}-x_{r 2}^{G}\right)+F \cdot\left(x_{r 3}^{G}-x_{r 4}^{G}\right)
\end{aligned}
$$

where $x_{\text {best }}$ is the best solution found so far in the current generation $G$.

\subsection{DE crossover operation}

The next stage that follows mutation in the DE flow cycle is crossover or vector perturbation operation to introduce the trial vectors/trial population $u_{i}(i=1,2, \ldots, N p)$. This operation is responsible for increasing the diversity among the parameters of target $x_{i}^{G}$ and $v_{i}^{G}$ donor vectors. Under this operation, the target and donor vectors exchange their components with respect to predefined conditions to form the trial vector $u_{i}^{G}$. DE family has two types of crossover - binary (uniform) crossover and exponential crossover. In what follows, the binary crossover is defined, which is also known as bin.

$$
u_{j, i}^{G}= \begin{cases}v_{j, i}^{G} & \text { if } \operatorname{rand}_{j, i}[0,1] \leq C R \quad \text { or } j=j_{\text {rand }} \\ x_{j, i}^{G} & \text { otherwise }\end{cases}
$$

where $\operatorname{rand}_{j, i}[0,1]$ is a uniform random number between 0 and $1 ; C R$ is a DE control parameter just like $F$. Its value determines the variation probability among the perturbed vectors. $C R \in[0,1+$ always takes positive 
value which cannot be greater than 1 (Price et al. 2005). $j_{\text {rand }} \in[1,2, \ldots, D]$ is the index of a randomly selected gene to ensure that the offspring $u_{i}^{G}$ inherits at least one component from the parent $v_{i}^{G}$.

\subsection{DE evaluation and selection operations}

In the final stage of DE, a greedy selection scheme takes place (see Eq. 8) to measure how far has been achieved for the best performance. This scheme is done by comparing the quality of the solution obtained from the crossover stage (trial vector $u_{i}^{G}$ ) with the quality of its corresponding target vector $x_{i}^{G}$ to determine who will survive to the next generation, $x_{i}^{G+1}(i=1,2, \ldots, N p)$. The quality of solution is calculated using the objective function $f$ (fitness function) designed for the problem in hand. This operation is described as

$$
x_{i}^{G+1}=\left\{\begin{array}{cc}
u_{i}^{G} & \text { if } f\left(u_{i}^{G}\right) \leq f\left(x_{i}^{G}\right) \\
x_{i}^{G} & \text { otherwise }
\end{array} \quad\right. \text { (for minimization problems) }
$$

From the above equation, if the trial vector has a lower or equal fitness function value, then it replaces the corresponding target point in the next generation; otherwise, the old point is retained in the next generation. This strategy guarantees that through generations the population will never deteriorate because the population either gets better (with respect to minimizing the fitness value) or remains the same.

\section{Adaptive Differential Evolution: Procedural analysis and comparison}

Generally, DE disposes three control parameters,

- Population size $(N p)$ : The total number of potential solutions in one generation

- Mutation factor $(F)$ : The amount of differentiation ratios that the perturbed solution can acquire

- Crossover rate $(C R)$ : The probability in which the offspring inherits the actual genes of a parent individual

The literature reveals many recent and prominent adaptive DE variants that show efficiency and reliability in their performance. In this study, notable adaptive DE algorithms (as listed in Fig. 3, there are 28 adaptive DE variants) have been reviewed and analyzed on a case-by-case basis according to a particular situation implemented (i.e. parameter control scheme and/or adaptive DE mutation strategy) within the new proposed taxonomy. Due to space limitation, 19 algorithms out of 28 have been thoroughly presented and discussed; the other 9 algorithms have been summarized in Table 8 . These 19 algorithms are as follows:

- FADE (Fuzzy Adaptive DE) is a parameter adaptive DE in which the control parameters $(F$ and $C R)$ of DE are adjusted using fuzzy logic (Liu and Lampinen 2005).

- $\mathbf{j D E}$ is a parameter adaptive DE in which the control parameters $(F$ and $C R$ ) of DE are adjusted using selfadaptive scheme (Brest et al. 2006).

- FiADE (Fitness-Adaptive DE) is a parameter adaptive DE that updates the values of $F$ and $C R$ during evolution based on the fitness function values of the individuals in the population (Ghosh et al. 2011).

- GADE (Greedy Adaptive Differential Evolution) is an adaptive DE algorithm with an effective greedy strategy that updates the values of $F$ and $C R$ during the run in a successive learning period (Leon and Xiong 2016).

- DESAP is a parameter adaptive DE in which the control parameters $(F, C R$ and $N p)$ of DE are all adjusted though evolution (Teo 2006). 
- JADE is a parameter adaptive DE in which the control parameters $(F$ and $C R)$ of DE are adjusted using selfadaptive learning scheme (Zhang and Sanderson 2009b).

- DEGL (DE with global and local neighborhoods) is a parameter adaptive DE with a novel mutation strategy. In this algorithm, new parameter control schemes that balance between the exploitation and exploration abilities in the DE mutation strategy during evolution is presented (Das et al. 2009b).

- $\quad \mathbf{M D E} \boldsymbol{\rho} \mathbf{B X}$ is a parameter adaptive DE in which the control parameters $(F$ and $C R)$ of DE are adjusted using self-adaptive learning scheme (Islam et al. 2012).

- $\quad \boldsymbol{p}$-ADE is a parameter adaptive DE that adjusts the parameters of $F$ and $C R$ and other control parameters related to its mutation scheme in an adaptive manner (Bi and Xiao 2011).

- SHADE (Success-History based Adaptive DE) is an improved version of JADE algorithm. It proposes an adaptation technique that controls the parameter settings of $F$ and $C R$ based on a historical memory of successful solutions and it is updated dynamically during the run. The advantage of the historical memory is to keep track of the control parameters values that generate the best solutions (Tanabe and Fukunaga 2013).

- L-SHADE is an extended version of SHADE algorithm in which a linear population size reduction (LPSR) is integrated to continually decrease the size of DE population using a linear function. This strategy has exhibited efficiency in saving the computation cost of the origin algorithm (Tanabe and Fukunaga 2014).

- EsDE ${ }_{r}$-NR (Ensemble sinusoidal differential evolution with niching reduction) is an enhanced version of the LSHADE-EpSin algorithm (L-SHADE with ensemble parameter sinusoidal adaptation) presented in (Awad et al. 2016). EsDE $\mathrm{r}_{\mathrm{r}} \mathrm{NR}$ features many significant adaptation merits in which two sinusoidal approaches and a Cauchy distribution are used as mixture to update the value of $F$ during evolution. In addition, the population size $N p$ has been reduced during generations using a novel niching-based reduction scheme. Finally, a restart method is activated at the late stage of evolution to further improve the quality of the solutions found so far (Awad et al. 2017a).

- $\quad \mathrm{SaDE}$ is a parameter and strategy adaptive DE in which the control parameters ( $F$ and $C R)$ of DE as well as the DE strategies are adjusted using adaptive techniques (Qin et al. 2009).

- EPSDE is a new version of adaptive DE in which an ensemble of control parameters and strategies are created then selected randomly for each individual (Mallipeddi et al. 2011).

- NRDE (Noise Resilient DE) is an improved DE algorithm with strategy and parameter control proposed for complex optimization problems, mainly, for functions corrupted with additive noise. This algorithm proposes three new mechanisms to achieve this objective: adaptive switching between two alternative mutation strategies, blending crossover, and threshold-based selection mechanism (Ghosh et al. 2017).

- SaDE-MMTS is a parameter and strategy adaptive DE in which the control parameters $(F$ and $C R)$ of DE as well as the DE strategies are adjusted using adaptive techniques. This algorithm is an integration of SaDE, JADE and local search algorithms (Zhao et al. 2011).

- SaM (SaJADE) is a parameter and strategy adaptive DE in which the control parameters $(F$ and $C R$ ) of DE as well as the JADE strategies are adjusted using adaptive techniques. This algorithm is an improvement of the JADE (Gong et al. 2011).

- EADE is an enhanced adaptive differential evolution algorithm that presents a novel mutation strategy and a new adaptive learning scheme to update the value of $C R$ during evolution. Both the mutation strategy and the adaptive scheme have successfully create the balance between the exploration and exploitation capabilities of DE and prove effectiveness in solving large-scale optimization problems (Mohamed 2017b).

- EFADE is an enhanced fitness-adaptive differential evolution algorithm with novel mutation. In this algorithm, a new triangular mutation strategy is presented to improve the exploration and exploitation capabilities of DE. In addition, two novel adaptation schemes for controlling the values of $F$ and $C R$ during evolution are suggested (Mohamed and Suganthan 2017). 
To convey the aforementioned information, this section is divided into THREE major subsections.

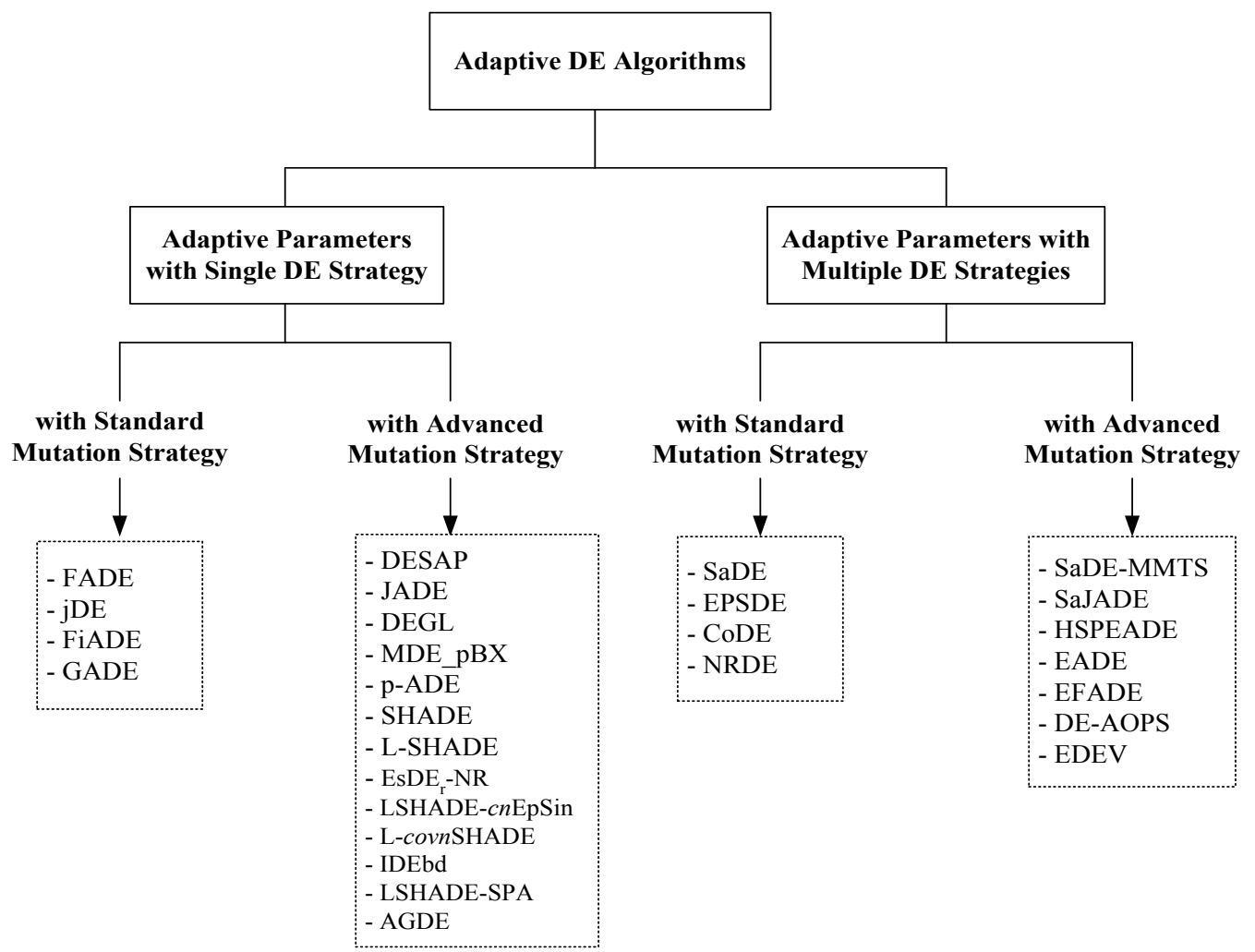

Fig 3: New classification illustrates the position of each adaptive DE variant with respect to the type of adaptive scheme(s) it applies

\subsection{DE with adaptive parameters and single strategy}

In this subsection, the main characteristics and mechanisms of 12 remarkable adaptive DE versions are stated in details on the basis of parameter adaptive schemes and DE strategies.

\subsubsection{Adaptive DE with single standard strategy}

\section{- FADE Algorithm}

FADE uses the standard DE scheme DE/rand/1/bin. It updates the values of $F$ and $C R$ in each generation using a mechanism, which is based on the fuzzy logic controller (FLC), whereby a fuzzy knowledge-based system is used to update the control parameters online in a dynamic adaptive manner to the inconsistent situation.

- The values of function values, population diversity $(F C)$, parameter vectors $(P C)$, and their updates after $n^{\text {th }}$ generations are calculated and then used as input to the FLCs. The values of the control parameters (i.e. $F$ and $C R$ ) are the outputs.

- The values of $F$ and $C R$ are then assigned to the fuzzy sets membership functions.

- ' $9 \times 2$ ' IF-THEN fuzzy rules statements are used to formulate the conditional statements that comprise fuzzy logic. 
- Mamdani's fuzzy inference method is used as the fuzzy control strategy to map from the given inputs to an output.

- Defuzzification is done to map from a space of fuzzy output to a space of real output.

\section{- jDE Algorithm}

$\mathrm{jDE}$ uses the standard DE strategy $\mathrm{DE} / \mathrm{rand} / 1 / \mathrm{bin}$. It updates the values of $F$ and $C R$ in a self-adaptive manner based on adjusting the control parameters $F$ and $C R$ by means of evolution and applied at the individual level. First, each individual $x^{G} \bar{i}^{0}(i=1,2, \ldots, N p)$ is associated with its corresponding control parameters $F_{i}$ and $C R_{i}$. In the first generation, these parameters are initialized to $F^{G}{ }_{i}^{0}=0.5$ and $C R^{G}{ }_{i}{ }^{0}=0.9$, then $F^{G+1}$ and $C R_{i}^{G+1}$ are assigned to values generated according to uniform distributions in $[0.1,1]$ and $[0,1]$ respectively during evolution as follows:

$$
\begin{aligned}
F_{i}^{G+1} & = \begin{cases}0.1+\text { rand }_{1} \times 0.9, & \text { if } \text { rand }_{2}<\tau_{1} \\
F_{i}^{G}, & \text { otherwise }\end{cases} \\
C R_{i}^{G+1} & = \begin{cases}\text { rand }_{3}, & \text { if rand } \\
C R_{i}^{G}, & \text { otherwise }\end{cases}
\end{aligned}
$$

where $\operatorname{rand}_{j} ; j \in\{1,2,3,4\}$ are uniform random values $\in[0,1]$. In this algorithm, $\tau_{1}$ and $\tau_{2}$ represent the probability limits that permit the adjustment of $F$ and $C R$ values, and they are both assigned to the same value 0.1 .

A recent study has been devoted to present a thorough statistical analysis to show the influence of tuning the randomness level parameter in the performance of jDE-like algorithms. The study has suggested values for the control parameters and the means to select them to better steer the iteration-temporal performance of the algorithms. Many open issues for the applications of the self-adaptive DE mechanisms into other mechanisms have also been indicated such as, in the online learning of ensemble strategies (Zamuda and Brest 2015).

\section{- FiADE}

In this algorithm, novel adaptation schemes for adjusting the values of $F$ and $C R$ online are proposed and then integrated with the standard DE/best/1/bin algorithm. The underlying idea behind these adaptive schemes is the best individual in the current generation will suffer from decreasing its step-size at the mutation stage to increase its chances of searching nearby the optimum area (neighborhood). At the crossover stage, the same individual will have its components propagated to its offspring more than the other individuals in the same population. Using these strategies creates an appropriate balance between the exploitation and exploration abilities of DE. FiADE is implemented with regard to the fitness function values of the latest individuals obtained, and this is the second attempt after (Ali and Törn 2004) who proposed a fitness-based adaptation scheme for $F$. Firstly, in FiADE, the following two different schemes (Scheme 1 and Scheme 2) are used to adjust the value of $F$ during evolution.

Scheme 1: $\quad F_{i}=F_{\max } \cdot\left(\frac{\Delta f_{i}}{\lambda+\Delta f_{i}}\right)$

where $\Delta f_{i}=\left|f\left(x_{i}\right)-f\left(x_{\text {best }}\right)\right|$ and $\lambda=\frac{\Delta f_{i}}{10}+10^{-4}$. Number $10^{-4}$ is added to avoid the problem of division by zero when $\Delta f_{i}=0$.

Scheme 2:

$$
F_{i}=F_{\max } \cdot\left(1-e^{-\Delta f_{i}}\right)
$$


In both schemes, $F_{\max }=0.8$ because this setting gives best results over various benchmark functions through experiments. The two equations above clearly show that when $\Delta f_{i} \rightarrow 0$ then $F_{i} \rightarrow 0$. For scheme $1, F_{i} \rightarrow 0.8$ when $\Delta$

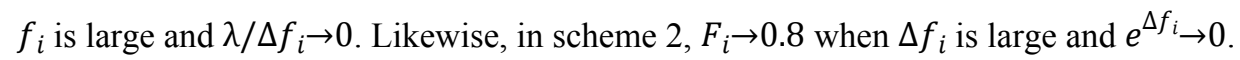

These two schemes play a key role in satisfying the objective of generating the best values of $F$ during evolution in a consecutive manner. Whenever the value of $\Delta f$ is greater than a threshold value (in the original paper (Ghosh et al. 2011) it is 2.4), scheme 2 will always have a greater value of $F$ to keep the exploration process active. On the contrary, when $\Delta f$ drops less than the value of the threshold, the search moves towards premature convergence as the value of $F$ decreases drastically. At this stage, scheme 1 is used to solve the degradation of the evolution as it decreases the value of $F$ at a lower rate, thus preventing the algorithm from falling into premature termination. Therefore, the adaptation scheme of $F$ can be formulated as

$$
F_{i}=\max \left(F_{1}, F_{2}\right)
$$

where $F_{1}=F_{\max } \cdot\left(1-e^{-\Delta f_{i}}\right)$ and $F_{2}=F_{\max } \cdot\left(\frac{\Delta f_{i}}{\lambda+\Delta f_{i}}\right)$.

The values of $C R_{i}$ have also been adapted in each generation based on the fitness values obtained for the donor vectors. As evidence of change, variable $\Delta f_{\text {donor }_{-} i}=\left|f\left(v_{i}\right)-f\left(x_{\text {best }}\right)\right|$ has been used as a measurement. If the value of $\Delta f_{\text {donor_ } i}$ is low, then the value of $C R$ should be higher because the donor vector is close to the best individual located in the current population, and thus it possesses good genetic components to be propagated to the offspring. On the contrary, when the value of $\Delta f_{\text {donor }_{-} i}$ is high, then the value of $C R$ should be lower. Another case is when the donor vector has better fitness value than even $x_{\text {best }}$ in the same population. In this case, the $C R_{i}$ should have been assigned to a very high value. Thus, the scheme for determining $C R_{i}$ can be formulated as

$$
\begin{aligned}
& \text { if } f\left(v_{i}\right) \leq f\left(x_{\text {best }}\right) \\
& \text { else } \quad C R_{i}=C R_{\text {const }} \\
& \\
& C R_{i}=C R_{\text {min }}+\frac{\left(C R_{\max }-C R_{\text {min }}\right)}{1+\Delta f_{\text {donor }_{-} i}}
\end{aligned}
$$

where $C R_{\text {const }}$ has been assigned to a high constant value of 0.95 . Eq. 14 clearly shows that when the value of $\Delta$ $f_{\text {donor }_{-} i} \rightarrow 0$, then $C R_{i}$ moves towards $C R_{\max }$ which is a high value and when $\Delta f_{\text {donor }_{-} i} \rightarrow \infty$, then $C R_{i}$ moves towards $C R_{\min }$ which is a lower value.

\section{- $G A D E$}

GADE algorithm proposes an effective greedy mechanism to dynamically update the values of $F$ and $C R$ during the evolution. The main idea of this adaptive strategy is that at every learning period (LP), the current $F$ and $C R$ values are compared with their neighboring setting values and then the settings with the best solution are selected. GADE algorithm has a very simple adaptation procedure that can be easily integrated with any DE strategy such as DE/rand/1. It starts with initializing the values of $F$ and $C R_{m}$ to 0.5 for both parameters. In each generation, the values of $F_{i}$ are selected randomly from the set $Z_{F}=\left\{F-d_{1}, F, F+d_{1}\right\}$, and the values of $C R_{i}$ are selected from the equation below.

$$
C R_{i}=\operatorname{rand}_{i}\left(\mu_{C R}, 0.2\right)
$$


where the value of the mean distribution, $\mu_{C R}$ is selected randomly from the set $Z_{C R}=$ $\left\{C R_{m}-d_{2}, C R_{m}, C R_{m}+d_{2}\right\}$. The two sets, $Z_{F}$ and $Z_{C R}$ represent the neighboring setting values. After a specified learning period $(G \% L P)$ the values of $F$ and $C R_{m}$ are then updated as follows:

$$
\begin{gathered}
F=\arg \max _{x \in Z_{F}} P R(x) \\
C R_{m}=\arg \max _{y \in Z_{C R}} P R(y)
\end{gathered}
$$

where $P R(x)$ and $P R(y)$ are the progress rates that are used as criteria to evaluate and compare the algorithm parameters assignments. $\mathrm{PR}$ is the average of the relative improvements (RI) from $N$ tests, using the $C$ assignment to produce trial solutions, and it is calculated as follows:

$$
\begin{gathered}
P R(C)=\frac{1}{N} \sum_{j=1}^{N} R I(C, j) \\
R I(C, j)=\left\{\begin{array}{c}
f\left(x^{k}\right) \cdot 10^{n}-f\left(v^{k}\right) \cdot 10^{n}, \quad \text { if } f\left(x^{k}\right) \geq f\left(v^{k}\right) \\
0,
\end{array} \quad\right. \text { otherwise }
\end{gathered}
$$

where $R I(C, j)$ is the relative improvement of the candidate assignment $C$ of either the scaling factor or crossover rate, $k$ is the test index, and $n$ is an integer number used to set the values of $f\left(x^{k}\right) \cdot 10^{n}$ and $f\left(v^{k}\right) \cdot 10^{n}$ within $[1,10]$ or $[-10,-1]$ ranges.

\subsubsection{Adaptive DE with single advanced strategy}

\section{- DESAP Algorithm}

\section{- Advanced DESAP Mutation and Crossover Schemes}

In DESAP the base strategy used is a bit different from the standard DE/rand/1/bin and of some sort similar to the strategy introduced in (Abbass 2002).

Crossover Scheme: The crossover operator is performed first with some probability, $\operatorname{rand}(0,1)<\delta_{r 1}$ or $i=j$, where $j$ is a randomly selected variable within individual $i$. The updating strategy is as follows,

$$
x_{\text {child }}=x_{r 1}+F \cdot\left(x_{r 2}-x_{r 3}\right)
$$

The ordinary amplification factor $F$ is set to 1 , thereby at least one variable in $x$ must be changed. Otherwise the value of $x_{\text {child }}$ and its control parameters will be set to the same values associated with $x_{r 1}$.

Mutation Scheme: The mutation stage is implemented with some mutation probability, $\operatorname{rand}(0,1)<\eta_{r 1}$; otherwise all the values will remain fixed.

$$
x_{\text {child }}=x_{\text {child }}+\operatorname{randn}\left(0, \eta_{r 1}\right)
$$

As can be seen from the equation above, DESAP mutation is not derived from any standard DE strategies.

\section{- DESAP Parameter Control Schemes}

DESAP not only updates the values of the mutation and crossover control parameters, $\eta$ and $\delta$, but, rather, it adjusts the population size $N p$ as well in a self-adaptive manner. All parameters undergo the evolution (i.e. 
crossover and mutation) in a way analogue to their corresponding individuals. The term $\delta$ has the same meaning as $C R$ and $\eta$ refers to the probability of applying the mutation scheme whereas the ordinary $F$ is kept fixed during the evolution process. Mainly, two versions of DESAP have been applied (Rel and Abs). The population size of both DESAP versions are initialized by generating, randomly, a population of size $(10 \times n)$ initial vectors; where $n$ denotes the number of design variables The mutation probability $\eta_{i}$ and crossover rate $\delta_{i}$ are both initialized to random values generated uniformly between $[0,1]$. The population size parameter $N p_{i}$ is initialized in DESAP-Abs version to,

$$
N p_{i}=\operatorname{round}(\text { initial population size }+\operatorname{randn}(0,1))
$$

whereas in DESAP-Rel to,

$$
N p_{i}=\operatorname{rand}(-0.5,0.5)
$$

The parameters $\delta, \eta$ and $\pi$ are updated at the same level with their corresponding individuals using the same crossover and mutation schemes (see Eq. 20-21).

Updating the crossover rate $\delta$

$$
\begin{gathered}
\delta_{\text {child }}=\delta_{r 1}+F \cdot\left(\delta_{r 2}-\delta_{r 3}\right) \\
\delta_{\text {child }}=\operatorname{randn}(0,1)
\end{gathered}
$$

Updating the mutation probability $\eta$

$$
\begin{gathered}
\eta_{\text {child }}=\eta_{r 1}+F \cdot\left(\eta_{r 2}-\eta_{r 3}\right) \\
\eta_{\text {child }}=\operatorname{randn}(0,1)
\end{gathered}
$$

Updating the population size $N p$

$$
\begin{aligned}
& \text { DESAP-Abs: } N p_{\text {child }}=N p_{r 1}+\operatorname{int}\left(F \cdot\left(N p_{r 2}-N p_{r 3}\right)\right) \\
& \text { DESAP-Rel: } N p_{\text {child }}=N p_{r 1}+\operatorname{int}\left(F \cdot\left(N p_{r 2}-N p_{r 3}\right)\right) \\
& \text { DESAP-Abs: } \quad N p_{\text {child }}=N p_{\text {child }}+\operatorname{int}(\operatorname{randn}(0.5,1)) \\
& \text { DESAP-Rel: } \quad N p_{\text {child }}=N p_{\text {child }}+\operatorname{randn}\left(0, \eta_{r 1}\right)
\end{aligned}
$$

The ordinary amplification factor $F$ is set to 1 . The evolution process of DESAP continues until it achieves a pre-specified population size $M$. Then, the new population size is calculated for the next generation as,

$$
\begin{array}{ll}
\text { DESAP-Abs: } & M_{\text {new }}=\operatorname{round}\left(\sum_{1}^{M} N p / M\right) \\
\text { DESAP-Rel: } & M_{\text {new }}=\operatorname{round}(M+(N p \times M))
\end{array}
$$

\section{- JADE Algorithm}

\section{- Advanced JADE Mutation Schemes}

Different mutation versions of JADE have been proposed in (Zhang and Sanderson 2009a) and (Zhang and Sanderson 2009b). The first new mutation scheme is called DE/current-to-pbest/1/bin (see Eq. 34), which has less greedy property than its previous specification scheme DE/current-to-best/1/bin, because it utilizes not only the information of the best individual but also the information of the $p \%$ good solutions in the current population. 


$$
v_{i}^{G}=x_{i}^{G}+F_{i \cdot}\left(x_{\text {best }}^{p, G}-x_{i}^{G}\right)+F_{i \cdot}\left(x_{r 1}^{G}-x_{r 2}^{G}\right),
$$

where $p \in(0,1]$ and $x_{\text {best }}^{p, G}$ is a random uniform vector chosen as one of the superior $100 p \%$ vectors in the current population. The second mutation scheme with an external archive is denoted as $A$, which is an archive introduced to store the recent explored inferior individuals that have been excluded from the search process. $A$ is first initialized to be empty. Thereafter, failed solutions in the selection operation of each generation are added to this archive. The new mutation operation is then reformulated as follows:

$$
v_{i}^{G}=x_{i}^{G}+F_{i \cdot}\left(x_{\text {best }}^{p, G}-x_{i}^{G}\right)+F_{i \cdot}\left(x_{r 1}^{G}-\tilde{x}_{r 2}^{G}\right),
$$

where $x_{i}^{G}$ and $x_{r 1}^{G}$ are generated from $P$ in the same way as in the original JADE, whereas $\tilde{x}_{r 2}^{G}$ is randomly generated from the union, $A \cup P$. Eventually, randomly selected solutions are going to be removed from the archive if its size exceeds a certain threshold to keep the archive within a specified dimension. Another JADE variant has been proposed to further increase the population diversity, and it is named archive-assisted DE/randto- $p$ best/ 1 as follows:

$$
v_{i}^{G}=x_{r 1}^{G}+F_{i \cdot}\left(x_{b e s t}^{p, G}-x_{r 1}^{G}\right)+F_{i \cdot}\left(x_{r 2}^{G}-\tilde{x}_{r 3}^{G}\right)
$$

\section{- JADE Parameter Control Schemes}

JADE updates four control parameters $\left(F, C R, \mu_{F}\right.$ and $\left.\mu_{C R}\right)$ during evolution.

Mutation factor $(F)$ and location parameter of mutation probability distribution $\left(\mu_{F}\right)$ : The mutation probability $F_{i}$ is independently generated in each generation for each individual $i$ according to the following formula:

$$
F_{i}=\operatorname{rand}_{i}\left(\mu_{F}, 0.1\right)
$$

where $\operatorname{rand}_{i}$ is a Cauchy distribution with location parameter $\mu_{F}$ and scale parameter 0.1 . If $F_{i} \geq 1$, then the value is truncated to be 1 or regenerated if $F_{i} \leq 0$. The location parameter $\mu_{F}$ is first initiated to be 0.5 . In this step, JADE shows some similarities in updating the mean of the distribution, $\mu_{C R}$ to the learning style used in Population Based Incremental Learning (PBIL) algorithm (Baluja 1994; Baluja and Caruana 1995). The standard version of the PBIL uses learning rate $L R \in(0,1]$ that must be fixed a priori. By utilizing Hebbianinspired rule, the difference rate $(1-L R)$ is then multiplied by the probability vector $(P V)$ that represents the combined experience of the PBIL throughout the evolution, whereas $L R$ is multiplied by each bit (i.e. gene's value) of the current individual(s) used in updating. Likewise, JADE updates the mutation distribution mean location, $\mu_{F}$. It is updated at the end of each generation after accumulating the set of all the successful mutation probabilities $F_{i}$ 's at generation $G$, denoted by $S_{F}$. The new $\mu_{C R}$ is updated as

$$
\mu_{F}=(1-c) \cdot \mu_{F}+c \cdot \operatorname{mean}_{L}\left(S_{F}\right),
$$

where $\operatorname{mean}_{L}($.$) is Lehmer mean,$

$$
\operatorname{mean}_{L}\left(S_{F}\right)=\frac{\sum_{F \in S_{F}} F^{2}}{\sum_{F \in S_{F}}{ }^{F}}
$$

Crossover probability $(C R)$ and mean of crossover probability distribution $\left(\mu_{C R}\right)$ : The crossover probability $C R_{i}$ is updated independently for each individual according to a normal distribution,

$$
C R_{i}=\operatorname{randn}_{i}\left(\mu_{C R}, 0.1\right),
$$


with mean $\mu_{C R}$ and standard deviation 0.1 and truncated to the interval $(0,1]$. The mean $\mu_{C R}$ is first initiated to 0.5. Then, $\mu_{C R}$ is updated in each generation after accumulating the set of all the successful crossover probabilities $C R_{i}$ 's at generation $G$, denoted as $S_{C R}$, thus calculate its mean ${ }_{A}\left(S_{C R}\right)$. The new $\mu_{C R}$ is updated as

$$
\mu_{C R}=(1-c) \cdot \mu_{C R}+c \cdot \operatorname{mean}_{A}\left(S_{C R}\right),
$$

where $c$ is a positive constant $\in(0,1]$ and $\operatorname{mean}_{A}(\cdot)$ is the usual arithmetic mean.

\section{- DEGL (DE with Global and Local Neighborhoods) Algorithm}

\section{- Advanced DEGL Mutation Strategy}

In this algorithm, a novel mutation strategy named Local and Global Neighborhood-Based Mutations is proposed. This new scheme has been inspired by the fact that a proper balance must be achieved in the search evolution between the two objectives, exploitation and exploration capabilities of the search technique. Two models have been used to achieve the aforementioned objectives: local neighborhood model and global mutation model. The local model is performed to generate a local donor vector $l_{i}^{G}$ of each individual in the current population as

$$
l_{i}^{G}=x_{i}^{G}+\alpha \cdot\left(x_{n \_b e s t_{i}}^{G}-x_{i}^{G}\right)+\beta \cdot\left(x_{p}^{G}-x_{q}^{G}\right)
$$

where $x_{n \_b e s t_{i}}^{G}$ is the best individual in the neighborhood of $x_{i}^{G}, x_{p}^{G}$ and $x_{q}^{G} ; p, q \in[i-k, i+k] ; p \neq q \neq i ; k$ is the neighborhood radius with value from 0 to $(N p-1) / 2$. This strategy satisfies the first objective (i.e. improve the local search). A global donor vector $g_{i}^{G}$ is also generated for each individual as

$$
g_{i}^{G}=x_{i}^{G}+\alpha \cdot\left(x_{g_{-} b e s t}^{G}-x_{i}^{G}\right)+\beta \cdot\left(x_{r 1}^{G}-x_{r 2}^{G}\right)
$$

where $x_{g_{-} b e s t}^{G}$ is the best individual in the entire current population; $r 1, r 2 \in[1, N P] ; r 1 \neq r 2 \neq i$. This strategy satisfies the second objective (i.e. improve the global search). In both models, $\alpha$ and $\beta$ are the scaling factors. These two models are then combined to formulate a general model that produces the actual donor individual as

$$
v_{i}^{G}=w \cdot g_{i}^{G}+(1-w) \cdot l_{i}^{G}
$$

where $w$ is a scalar factor with a value in $(0,1)$.

\section{- DEGL Parameter Control Schemes}

DEGL disposes four new control parameters $\alpha, \beta, w$, and $k$. However, the most effective parameter control among them is $w$ because it creates the balance between the exploitation and exploration capabilities in DEGL. The number of control parameters has been subsequently reduced because $\alpha=\beta=F$ has been considered. In DEGL, three adaptive schemes have been proposed to update the value of $w$.

1- Increasing Weight Factor: This scheme increases the value of $w$ from 0 to 1 whenever the search process requires. When the search moves towards exploration the value of $w$ is decreased to 0 . When the exploitation is required, the value of $w$ is increased towards 1 . For this reason, the initial vectors in the first generation are assigned to $w=0$, to activate the exploration moves; the contrary is applied at the final stages of the evolution in which exploitation is required. The middle value of $w, 0.5$ results in balanced performance in DEGL. Two schemes have been proposed for this purpose. 
Linear increment: $w$ is increased linearly from 0 to 1.

$$
w^{G}=\frac{G}{G_{\max }}
$$

Exponential increment: $w$ is increased exponentially from 0 to 1 .

$$
w^{G}=\exp \left(\frac{G}{G_{\max }} \cdot \ln (2)\right)-1
$$

2- Random Weight Factor: For each individual the value of $w$ is generated using uniformal random number, $w_{i}^{G}$ $\sim(0,1)$.

3- Self-Adaptive Weight Factor: As previously discussed in SECTION 3, when the value of the control parameter $w_{i}$ is embedded in the individual representation and undergoes evolution, this is known as selfadaptive setting, which is expressed as follows:

$$
\dot{w}_{i}^{G}=w_{i}^{G}+F \cdot\left(w_{g_{-} b e s t}^{G}-w_{i}^{G}\right)+F \cdot\left(w_{r 1}^{G}-w_{r 2}^{G}\right)
$$

where $w_{g_{-} b e s t}$ is the weigth factor associated with the best individual in the current population. $w_{i}^{G}$ are randomly initialized in the first generation to values in the range $(0.0,1.0)$ and then updated during generations.

\section{- MDE_pBX Algorithm}

\section{- Advanced MDE_pBX Mutation and Crossover Schemes}

Mutation Scheme: The new proposed mutation scheme DE/current-to-gr $r_{\text {bes }} / 1 /$ bin, utilizes the best individual $x_{\text {gr best }}^{G}$ chosen from the $q \%$ group of individuals that were randomly selected from the current population for each target vector. The group size $q$ of the MDE $\_$BX varies from $5 \%$ to $65 \%$ of the $N p$. The new scheme can be described as

$$
v_{i}^{G}=x_{i}^{G}+F_{i} \cdot\left(x_{g r_{\text {best }}}^{G}-x_{i}^{G}+x_{r 1}^{G}-x_{r 2}^{G}\right),
$$

where $x_{r 1}^{G}$ and $x_{r 2}^{G}$ are two different individuals randomly selected from the current population, and they are mutually different from the running individual $x_{i}^{G}$ and $x_{\text {gr }}^{G}{ }_{\text {best }}^{G}$.

Crossover Scheme: The new proposed recombination scheme $p$-Best has been defined as a greedy strategy, which is based on the incorporation of a randomly selected mutant vector perturbed by one of the $p$ top-ranked individual selected from the current population to yield the trial vector at the same index. Throughout evolution, the value of parameter $p$ is reduced linearly in an adaptive manner (see Eq. 55).

\section{- MDE pBX Parameters Control Schemes}

Modifications applied to the adaptive schemes in $M D E \_p B X$ : The scalar factor $F_{i}$ and crossover rate $C R_{i}$ of each individual are both altered independently in each generation using JADE schemes (see Eqs. 37 and 40). In MDE $p \mathrm{BX}$, both $\mu_{F}$ and $\mu_{C R}$ are subscribed to the same rule of adjusting. Firstly, their values are initialized to 0.5 and 0.6 respectively. Subsequently, they are updated in each generation as follows:

$$
\begin{gathered}
\mu_{F}=w_{F} \cdot \mu_{F}+\left(1-w_{F}\right) \cdot \text { mean }_{\text {pow }}\left(F_{\text {success }}\right) \\
\mu_{C R}=w_{C R} \cdot \mu_{C R}+\left(1-w_{C R}\right) \cdot \text { mean }_{\text {pow }}\left(C_{\text {success }}\right)
\end{gathered}
$$


where a set of successful scalar factors $F_{\text {success }}$ and a set of successful crossover probability $C R_{\text {success }}$ are generated from the current population. | | stands for the cardinality of each successful set. Variable $n$ is set to 1.5 then the mean power mean $_{\text {pow }}$ of each set is calculated as follows:

$$
\begin{gathered}
\operatorname{mean}_{\text {Pow }}\left(F_{\text {success }}\right)=\sum_{x \in F_{\text {success }}}\left(x^{n} /\left|F_{\text {success }}\right|\right)^{\frac{1}{n}} \\
\text { mean }_{\text {Pow }}\left(C R_{\text {success }}\right)=\sum_{x \in C R_{\text {success }}}\left(x^{n} /\left|C R_{\text {success }}\right|\right)^{\frac{1}{n}}
\end{gathered}
$$

including the calculation of the weight factors $w_{F}$ and $w_{C R}$,

$$
\begin{gathered}
w_{F}=0.8+0.2 \times \operatorname{rand}(0,1) \\
w_{C R}=0.9+0.1 \times \operatorname{rand}(0,1)
\end{gathered}
$$

the $\mu_{F}$ and $\mu_{C R}$ are formulized. As seen from Eqs. 53-54, the value of $w_{F}$ uniformly and randomly varies within the range $[0.8,1]$, whereas the value of $w_{C R}$ uniformly and randomly varies within the range $[0.9,1]$. The small random values used to perturb the parameters $\mu_{F}$ and mean $_{P o w}$ reveal an improvement in the performance of $\mathrm{MDE} \_\mathrm{BX}$ as it emphasizes slight variations on these two parameters each time $F$ is generated.

Crossover amplification factor $(p)$ : Throughout evolution, the value of parameter $p$ is reduced linearly in the following manner:

$$
p=\operatorname{ceil}\left[\frac{N p}{2} \cdot\left(1-\frac{G-1}{G_{\max }}\right)\right]
$$

where $\operatorname{ceil}(y)$ is the "ceiling" function that outputs the smallest integer $\geq y$. The reduction monotony of the parameter $p$ creates the required balance between the exploration and exploitation capabilities of DE.

\section{- p-ADE Algorithm}

\section{- Advanced p-ADE Mutation scheme}

A new mutation strategy called $\mathrm{DE} /$ rand-to-best/pbest/bin is used; which is, essentially, based on utilizing the best global solution and the best previous solution of each individual that are involved in the differential process, thus bringing in more effective guidance information to generate new individuals for the next generation. The detailed operation is as follows:

$$
v_{i}^{G}=W_{i}^{G} \cdot x_{r 1}^{G}+K_{i}^{G} \cdot\left(x_{\text {best }}^{G}-x_{i}^{G}\right)+F_{i}^{G} \cdot\left(x_{\text {pbesti }}^{G}-x_{i}^{G}\right)
$$

where $x_{\text {best }}^{G}$ denotes the best individual in the current generation $G . x_{r 1}^{G}$ is a random generated individual where $r$ $1 \in[1, N p]$ and $r 1 \neq i . x_{p b e s t i}^{G}$ denotes the best $i^{t h}$ previous individual picked up from the previous generation. The mutation's control parameters $W_{i}^{G}, K_{i}^{G}$, and $F_{i}^{G}$ of the $i^{\text {th }}$ individual are updated using a dynamic adaptive manner. The most remarkable merit of this mutation technique is the inclusion of three different working parts at the same time.

Inertial Part (Inheriting part) represented by $\left(W_{i}^{G} \cdot x_{r 1}^{G}\right)$ where the current individual, $v_{i}^{G}$ inherits traits from another individual at generation $G$.

Social Part (Learning Part) represented by $K_{i}^{G} \cdot\left(x_{\text {best }}^{G}-x_{i}^{G}\right)$ where the current individual, $v_{i}^{G}$ gains information from the superior individual in the current generation $G$. 
Cognitive Part (Private Thinking) represented by $F_{i}^{G} \cdot\left(x_{\text {pbesti }}^{G}-x_{i}^{G}\right)$ where the current individual, $v_{i}^{G}$ reinforces its own perception through the evolution process.

The high values of both the inertial and the cognitive part play a key role in intensifying the exploration searching space, thus improving its ability for finding the global solution. While the large values of the social part promotes connections among individuals, thus resulting to speed up the convergence rate. In $p$-ADE there is an additional mechanism which is called classification mechanism. This classification mechanism is coupled with the mutation scheme to be implemented on the whole population at each generation. Accordingly, the new mechanism divides the population's individuals into three classes:

Superior individuals: The first individuals' category where the fitness values of these individuals fall in the range $f_{i}-f_{\text {mean }}<-E\left(f^{2}\right)$, where $f_{\text {mean }}$ is the mean fitness values and $E\left(f^{2}\right)$ is the second moment of the fitness values of all individuals in the current generation. In this case, the exploration ability of the search process is enhanced by further intensifying the inertial and cognitive parts in order to increase the likelihood of the excellent individual to find the global solution in its neighborhood area. The corresponding individual is generated as follows:

$$
v_{i}^{G}=W_{i}^{G} \cdot x_{r 1}^{G}+F_{i}^{G} \cdot\left(x_{p b e s t i}^{G}-x_{i}^{G}\right)
$$

Inferior individuals: The second individuals' category where the fitness values of these individuals fall in the range $f_{i}-f_{\text {mean }}>E\left(f^{2}\right)$. The individual in this case has poor traits since its place in the search space is far away from the global optimum. Therefore, the exploration search ability is also intensified for rapid convergence rate. The corresponding individual is generated as follows:

$$
v_{i}^{G}=W_{i}^{G} \cdot x_{r 1}^{G}+K_{i}^{G} \cdot\left(x_{\text {best }}^{G}-x_{i}^{G}\right)
$$

Middle Individuals: The third individuals' category where the fitness values of these individuals fall in the range $-E\left(f^{2}\right)<f_{i}-f_{\text {mean }}<E\left(f^{2}\right)$. The individuals in this category are not superior nor are they inferior; therefore, the complete perturbation scheme (see Eq. 56) should be implemented entirely for further improving both the exploitation and exploration abilities.

\section{- $\quad$-ADE Parameter Control Schemes}

$p$-ADE comprises four control parameters involved in the search process, including three mutation scheme parameters $(W, F$ and $K$ ) and crossover rate $C R$. A dynamic adaptive scheme has been proposed to jointly update the four parameters through the run as follows:

$$
\begin{gathered}
W_{i}^{G}=W_{\text {min }}+\left(W_{\text {max }}-W_{\text {min }}\right) \times\left(\left(2-\exp \left(\frac{G}{G_{\text {max }}} \times \ln (2)\right)\right) \times \frac{1}{2}+\frac{f_{i}^{G}-f_{\text {min }}^{G}}{f_{\text {max }}^{G}-f_{\text {min }}^{G}} \times \frac{1}{2}\right) \\
K_{i}^{G}=K_{\text {min }}+\left(K_{\text {max }}-K_{\text {min }}\right) \times\left(\left(\exp \left(\frac{G}{G_{\text {max }}} \times \ln (2)\right)-1\right) \times \frac{1}{2}+\frac{f_{i}^{G}-f_{\text {min }}^{G}}{f_{\text {max }}^{G}-f_{\text {min }}^{G}} \times \frac{1}{2}\right) \\
F_{i}^{G}=F_{\text {min }}+\left(F_{\text {max }}-F_{\text {min }}\right) \times\left(\left(2-\exp \left(\frac{t}{G e n} \times \ln (2)\right)\right) \times \frac{1}{2}+\frac{f_{i}^{G}-f_{\text {min }}^{G}}{f_{\text {max }}^{G}-f_{\text {min }}^{G}} \times \frac{1}{2}\right) \\
C R_{i}^{G}=C R_{\text {min }}+\left(C R_{\text {max }}-C R_{\text {min }}\right) \times\left(\left(2-\exp \left(\frac{t}{G e n} \times \ln (2)\right)\right) \times \frac{1}{2}+\frac{f_{i}^{G}-f_{\text {min }}^{G}}{f_{\text {max }}^{G}-f_{\text {min }}^{G}} \times \frac{1}{2}\right)
\end{gathered}
$$


As seen from the above equations, the main adaptive scheme is equally captive to the influence of the number of generations achieved, as well as the fitness values. Technically, the value of each control parameter varies within its specified range, $W \in[0.1,0.9], K \in[0.3,0.9], F \in[0.3,0.9]$ and $C R \in[0.1,0.9]$ during the run.

\section{- SHADE Algorithm}

SHADE is an improved version of JADE algorithm specifically in updating the control parameter values of $F$ and $C R$. SHADE kept the mutation scheme of JADE w archive (see Eq. 35) as it is but modified the adaptation strategy of the $F$ and $C R$ mean values $\left(\mu_{F}\right.$ and $\mu_{C R}$ ). The main idea of SHADE is to no longer generate the values of $F_{i}$ and $C R_{i}$ from $\mu_{F}$ and $\mu_{C R}$ as two distribution mean values are updated once in each generation but are generated from a historical memory $\left(M_{F}, M_{C R}\right)$ that stores the mean values of $S_{F}$ and $S_{C R}$ each time these two sets are updated with new values of $\mu_{F}$ and $\mu_{C R}$, respectively. This new modification improves the performance of DE in general and JADE in specific due to the inclusion of more values in $M_{F, k}$ and $M_{C R, k}$ which leads the search towards different search regions especially when dealing with problems of different scenarios.

\section{- SHADE Parameter Control Schemes}

SHADE maintains a historical memory with $H$ entries for the mean values of $S_{F}$ and $S_{C R}$ and denoted as $M_{F, k}$ and $M_{C R, k}$, respectively, and $k \in[1, H]$. In the first generation, the values of all $M_{F, k}$ and $M_{C R, k}$ are initialized to 0.5 . When generating the values of $F_{i}$ and $C R_{i}$ in each generation, a new adaptation scheme is proposed as follows:

$$
\begin{gathered}
F_{i}=\operatorname{rand}_{i}\left(M_{F, r_{i}} 0.1\right) \\
C R_{i}=\operatorname{randn}_{i}\left(M_{C R, r_{i}} 0.1\right)
\end{gathered}
$$

where $r_{i}$ is randomly selected index within $[1, H]$. In case the values of $F_{i}$ and $C R_{i}$ exceed to 0 or 1 , the same adjusting procedure in JADE is followed. Before the end of each generation, the successful values of $F_{i}$ and $C R_{i}$ are stored in $S_{F}$ and $S_{C R}$, respectively. The values of these two sets are then used to update the historical memory of $M_{F}$ and $M_{C R}$ as follows:

$$
\begin{aligned}
M_{F, k}^{G+1} & = \begin{cases}\operatorname{meanw}_{L}\left(S_{F}\right) & \text { if } S_{F} \neq \emptyset \\
M_{F, k}^{G} & \text { otherwise }\end{cases} \\
M_{C R, k}^{G+1} & = \begin{cases}\operatorname{meanw}_{A}\left(S_{C R}\right) & \text { if } S_{C R} \neq \varnothing \\
M_{C R, k}^{G} & \text { otherwise }\end{cases}
\end{aligned}
$$

The value of $k$ is first initialized to 1 and then incremented by 1 each time an element is added to the memory. If $k$ reaches a threshold (it is $H$ in SHADE), then $k$ is set to 1 . The weighted Lehmer mean $\operatorname{meanw}_{L}\left(S_{F}\right)$ is computed using the equation below.

$$
\operatorname{meanw}_{L}\left(S_{F}\right)=\frac{\sum_{k=1}^{\left|S_{F}\right|} w_{k} \cdot S_{F, k}^{2}}{\sum_{k=1}^{\left|S_{F}\right|} w_{k} \cdot S_{F, k}}
$$

To avoid the bias towards small values that may be generated because of the arithmetic mean used in Eq. 41 , Peng et al. (2009) suggested using the following weighted mean (Peng et al. 2009): 


$$
\begin{gathered}
\operatorname{meanw}_{A}\left(S_{C R}\right)=\sum_{k=1}^{\left|S_{C R}\right|} w_{k} \cdot S_{C R, k} \\
w_{k}=\frac{\Delta f_{k}}{\sum_{k=1}^{\left|S_{C R}\right|} \Delta f_{k}}
\end{gathered}
$$

where $\Delta f_{k}=\left|f\left(u_{k}^{G}\right)-f\left(x_{k}^{G}\right)\right|$. Finally, in JADE the value of $p$ (see Eq. 34) is kept fixed during the run. In the SHADE algorithm, the value of this parameter is associated with each $x_{i}$ and altered during generations as follows:

where $p_{\min }=2 / N p$.

$$
p_{i}=\operatorname{rand}\left[p_{\min }, 0.2\right]
$$

\section{- L-SHADE Algorithm}

This algorithm is an improved version of SHADE algorithm in which a deterministic population resizing scheme is incorporated to dynamically reduce the size of population according to a linear function. This scheme is named as linear population size reduction (LPSR), and it is a specialized version of simple variable population sizing (SVPS) scheme proposed in (Laredo et al. 2009). The main concept of LPSR is to continually reduce the population size $N p$ linearly from the initial size which is $N p_{\text {init }}$ to the target population size which is $N p_{\min }$ using the function below.

$$
N p^{G+1}=\text { round }\left[\left(\frac{N p_{\text {min }}-N p_{\text {init }}}{M A X_{-} N F E}\right) \cdot N E F+N p_{\text {init }}\right]
$$

Where $M A X_{-} N F E$ is the maximum number of fitness evaluation, and $N F E$ is the current number of fitness evaluation. Based on the equation above, the worst ranking vectors $\left(N p^{G}-N p^{G+1}\right)$ are deleted whenever $N p^{G+1}<N p^{G}$.

L-SHADE has also suggested a slight improvement to the scheme of generating the values of $C R_{i}$, in which a terminal value " $\perp$ " is assigned to the $M_{C R, r_{i}}$ when $\max \left(S_{C R}\right)=0$ (i.e. all elements in $S_{C R}$ are 0 ). This modification has led to the following updated scheme:

$$
C R_{i}=\left\{\begin{array}{lc}
0 & \text { if } M_{C R, r_{i}=\perp} \\
\operatorname{randn}_{i}\left(M_{C R, r_{i}}, 0.1\right) & \text { otherwise }
\end{array}\right.
$$

The 0 value assigned to $C R_{i}$ enforces only one parameter change in the original crossover scheme of DE, which implies to slow down the convergence of the algorithm, and it is effective in the multimodal problems. Finally, the weighted Lehmer mean is used to calculate the mean values for both $S_{F}$ and $S_{C R}$ (i.e. $S$ ) sets using the equation below for both $M_{F}$ and $M_{C R}$.

$$
\begin{array}{r}
\operatorname{meanw}_{L}(S)=\frac{\sum_{k=1}^{|S|} w_{k} \cdot S_{k}^{2}}{\sum_{k=1}^{|S|} w_{k} \cdot S_{k}} \\
w_{k}=\frac{\Delta f_{k}}{\sum_{j=1}^{|S|} \Delta f_{j}}
\end{array}
$$




$$
\Delta f_{k}=\left|f\left(u_{k}^{G}\right)-f\left(x_{k}^{G}\right)\right|
$$

\section{- EsDE $E_{r}$-NR Algorithm}

In $\mathrm{EsDE}_{\mathrm{r}}-\mathrm{NR}$ algorithm, the advanced mutation strategy proposed in JADE is adopted (see Eq. 35 ). EsDE $\mathrm{r}_{\mathrm{r}}-\mathrm{NR}$ has three improved parts: ensemble sinusoidal parameter adaptation scheme, niching-based population size reduction, and restart method. They are presented briefly below.

\section{- $\quad E S D E_{r}$-NR Parameter Control Schemes}

As previously suggested in (Awad et al. 2016), in every generation in the first half of the evolution process $G \in\left[1, \frac{G_{\max }}{2}\right]$ the values of $F_{i}$ are updated using either one of the two sinusoidal adaptation approaches as presented below. The preference between these two approaches is implemented randomly.

sinusoidal decreasing adjustment

$$
\begin{aligned}
& F_{i}^{G}=\frac{1}{2} \cdot\left(\sin (2 \pi \cdot \text { freq } \cdot G+\theta) \cdot \frac{G_{\max }-G}{G_{\max }}+1\right) \\
& F_{i}^{G}=\frac{1}{2} \cdot\left(\sin \left(2 \pi \cdot \text { freq }_{i}^{G} \cdot G+\theta\right) \cdot \frac{G}{G_{\max }}+1\right)
\end{aligned}
$$

adjustment

where $\theta$ is the phase shift value, freq is set to a fixed value, and $f r e q_{i}^{G}$ is set automatically using an adaptive scheme as expressed below.

$$
\operatorname{freq}_{i}^{G}=\operatorname{rand}_{i}\left(\mu_{\text {freq }}, 0.1\right)
$$

Where $\mu_{\text {freq }}$ is the Cauchy distribution's mean with 0.1 as a standard deviation. The value of $\mu_{\text {freq }}$ is calculated using the usual arithmetic mean of the values in $S_{\text {freq }}$ set of the successful mean frequinces.

For the second half of the evolution, where $G \in\left[\frac{G_{\max }}{2}, G_{\max }\right]$, the values of $F_{i}$ are adapted in the same way suggested in (Tanabe and Fukunaga 2014) using Eq. 63. For the values of $C R_{i}$, they are adapted during the run using Eq. 64. Both $F$ and $C R$ values adopted in Eqs. 73-75 are also suggested L-SHADE to calculate the distribution mean values.

\section{○ EsDE $E_{r}$-NR Restart Method}

A restart mechanism is activated at the late stages of the evolution when the population size is reduced and the diversity of solutions is degraded. This mechanism is activated when the size of the population exactly reaches 20 individuals. Half of these individuals are then re-initialized using Eq. 1 and further modified using a Gaussian walk formula as follows:

$$
y_{i}=\operatorname{Gaussian}(\mu, \sigma)+\left(\operatorname{rand}(0,1) \cdot x_{\text {best }}-\operatorname{rand}(0,1) \cdot x_{i}\right)
$$

where the mean, $\mu$ is set to $x_{\text {best }}$ which is the best individual in the new generated group of individuals; $x_{i}$ is the $i^{\text {th }}$ individual in the same group of the new individuals; and $\sigma$ is computed as follows:

$$
\sigma=\left|\frac{\log (G)}{G} \cdot\left(x_{i}-x_{\text {best }}\right)\right|
$$

After these computations, a replacement occurs between the worst individuals and the new individuals in the $G_{L S}$ generations if the new individuals are better than the old individuals. 
After half of the function evaluations have been consumed, a novel population size reduction mechanism is activated to ensure that all individuals will have equal chance to evolve before the reduction is performed. From the start, each individual $x_{i}^{G}$ will have its Euclidian distance, $E D_{i}$ from the best individual, $x_{\text {best }}^{G}$ calculated as follows:

$$
E D_{i}=E D_{i}\left(x_{i}^{G}, x_{\text {best }}^{G}\right)=\sqrt{\left(x_{i, 1}^{G}-x_{\text {best }, 1}^{G}\right)^{2}+\left(x_{i, 2}^{G}-x_{\text {best }, 2}^{G}\right)^{2}+\ldots+\left(x_{i, D}^{G}-x_{\text {best }, D}^{G}\right)^{2}}
$$

These individuals are then sorted according to their $E D$ values and divided into two niche groups. The first niche contains the best individuals and the individuals in their neighborhood area (i.e. individuals with the smallest ED values). The second individual contains the remaining individuals. These two niches have equal size which is $\frac{N p}{2}$ , and since $N p$ is decreased after the first half of the evolution in each generation; the size of the two niches is also linearly decreased using Eq.71 in L-SHADE. The individuals in the second niche should be eliminated after satisfying different criteria.

\subsection{DE with adaptive parameters and multiple strategies}

In this subsection, the main characteristics and mechanisms of 7 adaptive DE versions are stated in detail on the basis of parameter adaptive schemes and multiple adaptive DE strategies.

\subsubsection{Adaptive DE with multiple standard strategies}

\section{- SaDE Algorithm}

- SaDE Adaptive Strategies

The main feature of SaDE is to automatically adapt multiple standard DE mutation strategies (DE/rand/1/bin, $\mathrm{DE} /$ rand-to-best/2/bin, $\mathrm{DE} / \mathrm{rand} / 2 /$ bin, and $\mathrm{DE} /$ current-to-rand/1 with no crossover) and update the corresponding control parameters during the evolution process using parameter, $p_{k}(k=1,2,3,4)$.

Determine the probability of applying each candidate strategy to the current population $\left(p_{k}\right)$ : Initially, the probabilities of applying each scheme $p_{k}^{G}$ is set to $1 / K$ to assign an equal probability for all strategies. The probability of applying each strategy is then updated every 50 generations in the following manner:

$$
p_{k}^{G}=\frac{S_{k}^{G}}{\sum_{k=1}^{K} S_{k}^{G}}
$$

where 


$$
S_{k}^{G}=\frac{\sum_{g=G-L P}^{G-1} n s_{k}^{g}}{\sum_{g=G-L P}^{G-1} n s_{k}^{g}+\sum_{g=G-L P}^{G-1} n f_{k}^{g}}+\varepsilon, \quad \text { for } k=1,2, \ldots, K ; G>L P
$$

where $K$ is the number of strategies available for perturbation. $L P$ is the period assigned for learning in which the learning process is activated only when $G>L P ; L P$ has been set to 50 generations. $n s_{k}^{G}$ (Success Memory) and $n f_{k}^{G}$ (Failure Memory) are both memories generated by the $k^{\text {th }}$ strategy and used to record the number of trial vectors that have succeeded or failed to enter the search process, respectively. The small value, that is, $\varepsilon=0.001$, is added to avoid the possibility of the null rate of success. Once the sizes of these memories reach a certain threshold, (i.e. after $L P$ iterations), all previous records will be eliminated from these memories, (i.e. $n s^{G-L P}$ and $n f^{G-L P}$ ) to allow those vectors that are generated in the current iteration to be stored. Finally, $S_{k}^{G}$ is divided by $\sum_{k=1}^{K} S_{k}^{G}$ to guarantee that the resultant $p_{k}^{G}$ is always summed to 1 .

\section{- SaDE Parameters Control Schemes}

Set the mutation factor $F_{i}$ values as independently generated in each generation according to Gaussian distribution with mean 0.5 and standard deviation 0.3 as follows:

$$
F_{i}=\operatorname{randn}(0.5,0.3)
$$

Accordingly, both the local (with small $F_{i}$ values) and global (with large $F_{i}$ values) search abilities will be kept throughout the evolution, hence to generate, good mutant vectors.

Crossover Probability $\left(C R_{i}\right)$ and the Mean Crossover Probability Distribution $\left(C R_{m}\right)$ : The strategy of controlling the crossover probability $C R$ is an adaptive controlled scheme. It starts with independently generating crossover probabilities $C R_{i}$ under Gaussian distribution with mean $C R_{m}$ and standard deviation 0.1 as follows:

$$
C R_{i}=\operatorname{randn}\left(C R_{m}, 0.1\right)
$$

The $C R_{i}$ values will remain fixed for five generations before the next generation has launched. Throughout these generations, $C R_{i}$ values that are associated with successful trial vectors are recorded. By contrast, the value of the median $C R_{m}$ is first initialized to 0.5 and then updated every 25 generations based on the successful $C R_{i}$ values as follows:

$$
C R_{m}=\frac{1}{K} \sum_{k=1}^{K} C R_{\text {suc }}(k),
$$

where $K$ denotes the number of successful $C R_{i}$ values accumulated over 25 generations, and $C R_{\text {suc }}$ is the $k^{\text {th }} C R$ successful value.

\section{- EPSDE Algorithm}

\section{- EPSDE Parameters Control Schemes and Strategies}

EPSDE is unlike other adaptive DE variants, it is an ensemble of mutation strategies and parameter values of DE. EPSDE does not involve a certain equation to modify the values of the control parameters. Rather, it 
assigns for each member of the initial population a mutation strategy randomly selected from a pool of mutation strategies with diverse characteristics and randomly takes values for the associated parameter from a pool of values. Throughout evolution, the population members that produce individuals better than the target vectors, their mutation strategies and associated parameter values are retained for the next generation, whereas those that fail to produce better individuals are reinitialized with a new mutation strategy and associated parameter values from the respective pools or from the successful combinations stored with equal probability. EPSDE comprises two pools.

Pool of mutation strategies: This pool includes the DE strategies that are involved in the evolution. These strategies have been selected with the following diverse characteristics:

1. Strategies relying on the best individual in the current population, $D E / b e s t / 2 /$ bin

2. Strategies bearing strong exploration capabilities, $\mathrm{DE} / \mathrm{rand} / 1 / \mathrm{bin}$

3. Strategies being rotational invariant without crossover, $\mathrm{DE} /$ current-to-rand/1

Pool of parameter control values: In this pool, the two crucial parameter values $(F$ and $C R$ ) are set to different ranges. The pool of $C R$ values is taken in the range $0.1-0.9$ in steps of 0.1. The pool of $F$ values is taken in the range $0.4-0.9$ in steps of 0.1 .

\section{- NRDE Algorithm}

In NRDE algorithm different mechanisms have been proposed to adapt the parameter control values of $F$ and $C R$ as well as the two standard mutation strategies of DE (DE/rand/1 and DE/best/1). The work proposed in NRDE is an extended version of a published work (Das et al. 2015) and as a new attempt after few attempts on investigating the performance of DE on strongly noisy problems such as the one presented in (Caponio and Neri 2009).

\section{- NRDE Parameters Control Schemes}

The control parameters $F$ and $C R$ of NRDE are altered using a simple mechanism that requires no prior knowledge or feedback from the search space during the run such as learning strategy. For each offspring to be generated, the value of $F$ is selected randomly from the set of feasible values $\{0.5,2\}$. At the same time, the value of $C R$ is selected randomly from the interval $[0,1]$ for each donor vector to be generated.

\section{- NRDE Adaptive Mutation Strategies}

NRDE proposes a simple mechanism to switch between two alternative DE mutation strategies (DE/rand/1 and $\mathrm{DE} /$ best/1) without using a pool of strategies. The selection between these two strategies is implemented in an equally probabilistic switch which is 0.5 . The reason behind the use of these two strategies is DE/rand/1 is known as a very effective strategy in creating population with high diversity (i.e. highly explorative), whereas $\mathrm{DE} / \mathrm{best} / 1$ focuses on creating solutions by perturbing the best solutions found so far in various search directions (i.e. highly exploitative).

\section{- NRDE Adaptive Crossover Strategies}

Using the same switching manner in mutation strategies, NRDE proposes a simple mechanism to switch between two alternative DE crossover strategies in an equally probabilistic manner which is 0.5 . One of these strategies is newly proposed in this algorithm as follows: 


$$
u_{j, i}^{G}=\left\{\begin{array}{cc}
b \cdot x_{j, i}^{G}+(1-b) \cdot v_{j, i}^{G} & \text { if } \text { rand }_{j, i} \leq 0.5 \text { or } j=j_{r} \\
x_{j, i}^{G} & \text { othewise }
\end{array}\right.
$$

where the value of parameter $b$ is chosen randomly from three values $0.2,0.5,0.9$. The main reason behind the use of these three values is because when $b$ is small (i.e. 0.2), a large portion relative to the donor vector is used. When $b$ is in the middle (i.e. 0.5), an equal portion is taken from both the target and donor vector. Finally, when the value of $b$ is 0.9 , a gene component is given very near to the target vector. The second crossover strategy is the standard DE crossover defined in Eq.7.

\section{-NRDE and Self-Adaptive Individual Selection Strategy}

NRDE adopted three cases in the survival selection step instead of two as in most of DE variants because the main objective of NRDE is to tackle noise or uncertainties in the optimization problems, and the standard greedy selection strategy may fail to distinguish whether the selected solution is absolutely superior or inferior in comparison to its parent. These cases are defined as follows:

$$
x_{i}^{G+1}= \begin{cases}u_{i}^{G}, & \text { if } \frac{f\left(u_{i}^{G}\right)}{f\left(x_{i}^{G}\right)} \leq 1, \\ u_{i}^{G}, & \text { if } \frac{f\left(u_{i}^{G}\right)}{f\left(x_{i}^{G}\right)} \leq 1+\delta, \\ x_{i}^{G}, & \text { otherwise }\end{cases}
$$

where $\delta$ is adapted in a self-adaptive manner using the following formula:

$$
\delta^{G+1}=\delta^{G} \cdot(1-\alpha)^{i}
$$

where $i$ is the generation index, and the value of $\alpha$ is very small which is $0.04 . \delta$ is initially set to 0.3 .

\subsubsection{Adaptive DE with multiple advanced strategies}

\section{- SaDE-MMTS Algorithm}

SaDE-MMTS has been proposed to enhance the performance of the standard SaDE algorithm; by incorporating SaDE with the JADE mutation strategy and integrating it with the modified multi-trajectory algorithm (MMTS) to solve problems with complex characteristics and high dimensionality. This integration can be encapsulated into three main parts: SaDE-MMTS = JADE mutation scheme + SaDE algorithm + MMTS method (Local Search), as follows:

\section{- SaDE-MMTS Advanced Adaptive DE Strategies}

JADE mutation strategy with external archive (see Eq. 35) is adopted and engaged with three crossover operators (binomial and exponential), and no crossover option as well, to generate the trail vectors for the new population. The expected number of perturbation strategies is three, and they are applied according to the strategy probability as in the SaDE algorithm. The selection of the mutation strategy is according to the probability $p_{k}^{G}$ of applying each JADE with archive strategy in the current population (see Eq. 82).

- SaDE Parameters Control Schemes 
The control parameters $F$ and $C R$ are updated through evolution in the same manner used in SaDE (see Eqs. 83-84).

\section{- MMTS method}

The original multi-trajectory (MTS) algorithm (Tseng and Chen 2007; Tseng and Chen 2008) is first proposed to solve large scale global optimization problems. The main underlying idea of this algorithm is the employment of randomly selected search combinations (i.e. agents) uniformly distributed over the whole search space to seek out for better solutions. Each combination applies one of the three candidate local search methods that better fits the search space characteristics of a solution's neighborhoods. These combinations are generated using the basic orthogonal array $O A_{n \times k}$, where $n$ is the number of testing experiments and $k$ is the number of factors in each experiment.

\section{- $\quad S a M$}

SaM is a strategy adaptation mechanism that can be integrated with any DE variant to make it strategy adaptive. SaM creates a pool of strategies and selects the candidate strategy to be applied on the running individual $x_{i}$ from this pool according to Eq. 89 .

$$
S_{i}=\left\lfloor\eta_{i} \times K\right\rfloor+1
$$

where $\eta_{i} \in[0,1)$ is a strategy parameter control variable. $K$ is the total number of strategies in the pool and $S_{i}$ $=1,2, \ldots, K$ the selected DE strategy. For example, suppose $K=4$ and at a certain generation $\eta_{i} \in[0,0.25)$, then based on the calculation of Eq. 89 the value of $S_{i}$ is 1 .

SaM has suggested three approaches to update the value of $\eta_{i}$ during evolution. In this study, the first approach has been considered which is inspired by the parameter adaptation equation of JADE. For each individual $x_{i}$ at generation $G$, a new value for $\eta_{i}$ is generated as follows:

$$
\eta_{i}=\left\{\begin{array}{lc}
\operatorname{randn}_{i} & G=1 \\
\operatorname{randn}_{i}\left(\mu_{s}, 0.1\right) & \text { otherwise }
\end{array}\right.
$$

where $\operatorname{randn}_{i}\left(\mu_{s}, 0.1\right)$ indicates a normal random distribution of mean $\mu_{s}$ and standard deviation 0.1 . The mean $\mu_{s}$ is initialized to be 0.5 and then updated at the end of each generation in the same adaptation equation used in JADE (see Eq. 41) as follows:

$$
\mu_{s}=(1-c) \times \mu_{s}+c \times \operatorname{mean}_{A}\left(H_{S}\right)
$$

where $H_{s}$ denotes as the set of all successful DE strategy parameters $\eta_{i}$ 's at generation $G$.

SaM mechanism has been applied on JADE strategies to create a new approach called SaJADE. SaJADE employed a pool of different JADE strategies: (1) DE/current-to- $p$ Best/1/bin with no archive; (2) DE/current-to$p$ Best/1/bin with archive; (3) DE/rand-to- $p$ Best/1/bin with no archive; (4) DE/rand-to- $p$ Best/1/bin with archive. The parameter adaptive equations used to update the values of $F_{i}$ and $C R_{i}$ are also JADE schemes as in Eq. 3741. For updating the value of $C R_{i}$, all strategies use Eq. 40. The difference is in updating the value of $F_{i}$ in which strategies 1 and 3 use Eq. 37; whereas strategies 2 and 4 use a modified version of Eq. 37 which is normal distribution generator as follows:

$$
F_{i}=\operatorname{randn}_{i}\left(\mu_{F}, 0.1\right)
$$

\section{- EADE Algorithm}

- Advanced Adaptive EADE Mutation scheme 
This algorithm proposes a novel mutation strategy that contributes to balance the tendency of both the exploration and exploitation capabilities of DE, as well as enhancing its convergence rate. This new strategy has two aims to guide the search process towards fine evolution. The first aim is to preserve the exploration capability during evolution and avoid premature convergence at local optima by preventing the individuals from not always following the best individual in the current population. The second is to preserve at the same time the exploitation capability by preventing the search process from following the bottom worst individual, thus, guiding the search towards promising sub-regions in the search space. These two objectives can be met by applying the new mutation strategy as follows:

$$
v_{i}^{G+1}=x_{r}^{G}+F_{1} \cdot\left(x_{p_{-} \text {best }}^{G}-x_{r}^{G}\right)+F_{2} \cdot\left(x_{r}^{G}-x_{p_{-} w o r s t}^{G}\right)
$$

where $x_{r}^{G}$ is randomly chosen individual from the middle $(N p-2(100 p \%))$ of the current population; $x_{p_{-} \text {best }}^{G}$ is a randomly chosen individual from the top $100 p \%$ individuals; $x_{p_{-} w o r s t}$ is a randomly chosen individual from the worst $100 p \%$ individuals. In both cases, $p \in(0,1]$; and $F_{1}$ and $F_{2}$ are uniformly and independently generated in the range $(0,1)$. From the equation above, sharing information from the best to worst individuals in the same population helps direct the search to different sub-regions in the search space by balancing the local exploitation and global exploration tendencies in the DE population. In EADE, this new mutation strategy is applied with probability of 0.5 in which the standard DE/rand/1/bin is applied otherwise.

\section{- EADE Parameter Control Schemes}

EADE proposes a novel adaptive scheme for gradual change of the values of $C R_{i}$ that can take benefit from the experience of the individuals in the search space during evolution which, in turn, can considerably balance the common trade-off between the population diversity and convergence speed. This scheme can be implemented by applying a simple and straightforward procedure. This procedure has specific parameters to be defined and set to values before and during the run. The first parameter is the pool $A=$ $[0.05,0.1,0.2,0.3,0.4,0.5,0.6,0.7,0.8,0.95]$ of pre-specified values for $C R$. These values are selected randomly from $A$ for each $C R_{i}$, using uniform distribution during evolution. $A$ is updated during and after a predefined learning period $\left(L P=10 \% G_{\max }\right)$. Another parameter is $C_{-} R_{-}$lag_List $_{i}$, which is a flag that is set to either 0 or 1 to indicate whether the target vector is better than the trial vector or the opposite, respectively. Failure_counter_list ${ }_{i}$ is a parameter used to monitor the progress of the individuals' fitness values after $L P$ completion. If no improvement is observed in the fitness, then the counter of that individual is increased by 1 . This process is repeated until Max_failure_counter $=20$ is reached. Another important parameter is CR_Ratio_List $k_{k}$ which is used to record the relative change improvement ratio (RCIR) for each $C R_{k}$ in $\mathrm{A}$ as

$$
\text { CR_Ratio_List }{ }_{k}=C R_{-} \text {Ratio_List }_{k}+\left(1-\frac{\min \left(\left|f\left(u_{i}^{G+1}\right)\right|,\left|f\left(x_{i}^{G}\right)\right|\right)}{\max \left(\left|f\left(u_{i}^{G+1}\right)\right|,\left|f\left(x_{i}^{G}\right)\right|\right)}\right)
$$

Equation 94 is implemented only when the CR_Flag_List ${ }_{i}=1$, which is the case where the trial vector is better than the target vector. The complete pseudocode of EADE is available in (Mohamed 2017b) in Figs. 2 and 3.

\section{- EFADE Algorithm}

- Advanced EFADE Mutation Scheme 
EFADE proposed a novel mutation strategy coined as triangular mutation. Its main concept is to enhance the balance between the global exploration and local exploitation tendencies of DE by investing vectors with different properties in the same mutation rule. These vectors are as follows:

- $\quad x_{\text {best }}, x_{\text {better }}$ and $x_{\text {worst }}$ are three random individuals selected from the current population ordered based on tournament selection.

- $\quad \bar{x}_{c}^{G}$ is called a convex combination vector of the triangle (three randomly selected vectors combined in the form of linear equation) as:

$$
\bar{x}_{c}^{G}=w_{1}^{*} \cdot x_{\text {best }}+w_{2}^{*} \cdot x_{\text {better }}+w_{3}^{*} \cdot x_{w o r s t}
$$

where $w_{i}^{*} \geq 0$ and $\sum_{i=1}^{3} w_{i}^{*}=1 ; w_{i}^{*}$ are called the real weights and are calculated using $w_{i}^{*}=w_{i} /$ $\sum_{i=1}^{3} w_{i}, i=1,2,3 . w_{i}$ is calculated using the following equation:

$$
w_{i}=1-\left|\frac{f\left(x_{\text {best }}\right)-f\left(x_{i}\right)}{f\left(x_{\text {best }}\right)-f\left(x_{\max }\right)}\right|, \quad i=1,2,3
$$

As a minimization problem, $f\left(x_{\text {best }}\right)=f\left(x_{\min }\right)=\min \left\{f\left(x_{i}\right)\right\}, i=1,2,3$ is the individual with the minimum fitness value among the three randomly selected vectors. $f\left(x_{\max }\right)$ is the individual with maximum fitness value in the current population.

Using the vectors above, the new mutation scheme can be formulated as follows:

$$
v^{G+1}=\bar{x}_{c}^{G}+F_{1} \cdot\left(x_{\text {best }}^{G}-x_{\text {better }}^{G}\right)+F_{2} \cdot\left(x_{\text {best }}^{G}-x_{\text {worst }}^{G}\right)+F_{3} \cdot\left(x_{\text {better }}^{G}-x_{\text {worst }}^{G}\right)
$$

where $F_{1}, F_{2}$ and $F_{3}$ are the mutation scalar factors that are generated in each generation using the new adaptation scheme. The mutation scheme above is implemented interchangeably with $\mathrm{DE} / \mathrm{rand} / 1$ scheme with respect to a nonlinear probability condition as

$$
\begin{aligned}
& \text { If }\left(\operatorname{rand}(0,1) \geq\left(1-\frac{G}{G_{\max }}\right)^{2}\right) \text { Then } \\
& \qquad v_{i}^{G+1}=\bar{x}_{c}^{G}+F_{1} \cdot\left(x_{\text {best }}^{G}-x_{\text {better }}^{G}\right)+F_{2} \cdot\left(x_{\text {best }}^{G}-x_{\text {worst }}^{G}\right)+F_{3} \cdot\left(x_{\text {better }}^{G}-x_{\text {worst }}^{G}\right)
\end{aligned}
$$

Else $\quad v_{i}^{G+1}=x_{r 1}^{G}+F \cdot\left(x_{r 2}^{G}-x_{r 3}^{G}\right)$

where $\operatorname{rand}(0,1)$ is a uniform random number generator between 0 and $1, i \neq r 1 \neq r 2 \neq r 3$ are indices randomly selected from the current population, and $F$ is set to 0.7 .

\section{- EFADE Parameter Control Schemes}

EFADE proposes two adaptation schemes to update the values of $F$ and $C R$ during evolution. These new schemes have the advantage of not imposing extra parameters or determining a learning period while adapting the control parameters.

Scalar factor adaptive scheme: The basic concept of this adaptation scheme is how to dynamically maintain a proper balance between the local exploitation and global exploration aspects during generations. For this 
purpose, three different scalar factors $\left(F_{1}, F_{2}\right.$ and $\left.F_{3}\right)$ have been involved in the new mutation scheme. The values of these parameters are updated in each generation as follows:

$$
F_{i}=\operatorname{rand}\left(0, k_{i}\right), \quad i=1,2,3
$$

where

$$
\begin{aligned}
& k_{1}= \begin{cases}\left|\frac{f\left(x_{\text {better }}\right)}{f\left(x_{\text {best }}\right)}\right|+\varepsilon, & \text { if }\left|\frac{f\left(x_{\text {better }}\right)}{f\left(x_{\text {best }}\right)}\right|<1 \\
\left|\frac{f\left(x_{\text {best }}\right)}{f\left(x_{\text {better }}\right)}\right|+\varepsilon, & \text { otherwise, }\end{cases} \\
& k_{2}= \begin{cases}\left|\frac{f\left(x_{\text {worst }}\right)}{f\left(x_{\text {best }}\right)}\right|+\varepsilon, & \text { if }\left|\frac{f\left(x_{\text {worst }}\right)}{f\left(x_{\text {best }}\right)}\right|<1 \\
\left|\frac{f\left(x_{\text {best }}\right)}{f\left(x_{\text {worst }}\right)}\right|+\varepsilon, & \text { otherwise, }\end{cases} \\
& k_{3}= \begin{cases}\left|\frac{f\left(x_{\text {worst }}\right)}{f\left(x_{\text {better }}\right)}\right|+\varepsilon, & \text { if }\left|\frac{f\left(x_{\text {worst }}\right)}{f\left(x_{\text {better }}\right)}\right|<1 \\
\left|\frac{f\left(x_{\text {better }}\right)}{f\left(x_{\text {worst }}\right)}\right|+\varepsilon, & \text { otherwise, }\end{cases}
\end{aligned}
$$

where $\operatorname{rand}\left(0, k_{i}\right)$ is a uniform random generator between 0 and $k_{i}$, and $\varepsilon=0.0001$ is a small number added to avoid zero value which implies no perturbation. If $k_{i}>1$, then its value is truncated to 1 . This adaptation scheme of $F_{1}, F_{2}$ and $F_{3}$ have successfully achieved the desired balance of the two contradictory aspects by controlling the amplification of the perturbation of the mutation scheme through generations.

Crossover rate adaptive scheme: The main idea of this scheme is to select an appropriate control value for the $C R$ of each target vector from recommended setting values in (Rönkkönen et al. 2005), distributed into two sets. Then, the selection of these values is updated using learning strategy, depending on their experiences of producing promising vectors through generations as follows:

If $G=1$ then

$$
C R_{i}^{1}= \begin{cases}C R_{1}, & \text { if rand }(0,1) \leq 0.5 \\ C R_{2}, & \text { otherwise }\end{cases}
$$

Else

$$
C R_{i}^{G}= \begin{cases}C R_{1}, & \text { if } \operatorname{rand}(0,1) \leq p_{1} \\ C R_{2}, & \text { if } p_{1}<\operatorname{rand}(0,1) \leq p_{1}+p_{2}\end{cases}
$$

where $C R_{1} \in[0.05,0.15]$ and $C R_{2} \in[0.9,1]$ are the two suggested sets, $p_{j}, j=1,2, \ldots, m$ is the probability of selecting the $C R$ value from set $j$ which is first initialized to $1 / j$, and $m=2$ is the total number of sets. $p_{j}$ is dynamically updated during generations using the following equation:

$$
\begin{gathered}
p_{j}^{G+1}=\left((G-1) \cdot p_{j}^{G}{ }_{j}^{1}+p s_{j}^{G}\right) / G, \\
p s_{j}^{G}=\frac{s_{j}^{G}}{\sum_{j=1}^{m} s_{j}^{G}},
\end{gathered}
$$

where 


$$
s_{j}^{G}=\frac{n s_{j}^{G}}{\sum_{g=1}^{G} n s_{j}^{g}+\sum_{g=1}^{G} n f_{j}^{g}}+\varepsilon,
$$

where $p s_{j}^{G}$ is the probability of selecting the $j^{\text {th }}$ set, $s_{j}^{G}$ is the success ratio of the individuals who successfully survived to the next generation, $n s_{j}^{G}$ and $n f_{j}^{G}$ are two counters for the individuals who survived or failed in the selection operations, respectively, and $\varepsilon=0.01$ is a very small number to avoid the zero value of $s_{j}^{G}$. Arguably, the small values of $C R$ have exert effects at the early stage of evolution because of the high diversity the first populations always have. By contrast, in the final generations, the large values of $C R$ can increase the diversity of population as the individuals will all be close to the best individual.

\subsection{Discussion on adaptive DE variants}

Nineteen adaptive DE algorithms have been presented in the previous subsections. These algorithms clearly exhibit diversity in terms of characteristic, structure, complexity and algorithmic logic. Despite their advantages, these algorithms show shortcomings in some particular cases. In this subsection, comparisons have been established among these methods according to the taxonomy provided in SECTION 3, their algorithmic design similarities and differences and pros and cons of each algorithm are provided in Tables 2-7. In addition to these $19 \mathrm{DE}$ variants, another 9 important and recent DE algorithms have been summarized in Table 8 to offer more adaptive algorithmic design insights. Finally, an estimated rank of these algorithms has been presented in Fig. 4.

\subsubsection{Adaptive DE conceptual similarities and differences}

In this subsection, we discuss how the aforementioned methods relate and differ from one another and from the standard DE algorithm based on mutation strategy and parameter control schemes used in each algorithm.

\section{- Comparison-based DE mutation scheme}

The 19 algorithms presented under this comparison differ in their mutation strategies, as seen in Table 2, which summaries the mutation schemes employed in each algorithm and categorized according to the taxonomy provided in SECTION 3. From Table 2, we can observe that the most common mutation strategy is DE/current-to-pbest/ 1 with and without archive. This new strategy has been adopted in many DE algorithms such as JADE, SHADE, L-SHADE and EsDE $\mathrm{r}_{\mathrm{r}}$-NR algorithms because this strategy has proved effective performance. All the algorithms adopt the classical crossover (bin) and the standard selection operations in their main work except DESAP, which uses a modified crossover scheme similar to that of $\mathrm{DE} / \mathrm{rand} / 1$ mutation scheme, MDE $p \mathrm{BX}$, which uses a new modified crossover scheme called $p$-Best, and NRDE that uses a simple mechanism to switch between two crossover schemes, one of these schemes is newly proposed in NRDE; while SaDE, EPSDE, NRDE, SaDE-MMTS, SaJADE, EADE and EFADE invent new adaptive schemes that can make a selection among a pool of candidate mutation schemes. In addition, SaDE-MMTS uses three crossover aspects (binomial, exponential and no crossover) integrated with their corresponding mutation strategies creating a pool of DE strategies to automatically adapt one of them.

\section{- Comparison-based DE parameter control schemes}

The comparison in this subsection is based on the parameter setting taxonomy presented in SECTION 3. Table 3 elucidates the main features of the adaptation scheme used to control the three main control parameters $(F, C R, N p)$ in the 19 adaptive DE variants. This table shows how the adaptive concept is 
revealed by applying an adaptive rule to at least one of the algorithm components. From the same table, none of the algorithms has considered adapting the population size $N p$ except DESAP, L-SHADE and EsDE $_{\mathrm{r}}$-NR algorithms. The focus is only on $F$ and $C R$.

\subsubsection{Adaptive DE strengths and drawbacks}

In this subsection, conceptual strengths and drawbacks of the 19 algorithms have been discussed in terms of modifying the main DE strategies, additional components that have been included in the original DE algorithm and function as adaptive features. All the modifications and integrations proposed include extra moves in the original DE as well as create the proper balance between the exploitation and exploration characteristics. However, drawbacks need to be considered.

\section{- Comparison-based DE mutation strategy}

In this subsection, the $19 \mathrm{DE}$ algorithms have been compared based on the mutation scheme used in each algorithm. Tables 4 and 5 illustrate the comparison of algorithms in terms of pros and cons of each mutation strategy.

\section{- Comparison-based DE parameter control schemes}

Tables 6 and 7 elucidate the points of strengths and drawbacks of the adaptation scheme used to control the three main parameters $(F, C R$ and $N p)$ in the $19 \mathrm{DE}$ variants. The two tables indicate common pros and cons among certain algorithms because they use the same adaptation scheme(s).

\subsubsection{Supplemental adaptive DE variants}

Table 8 gives a summary of 9 important and very recent adaptive DE algorithms each according to its mutation strategy or ensemble of strategies it utilizes and the adaptive scheme(s) it employs to adjust the values of $N p, F$ and $C R$ control parameters. This table gives us an indication that the L-SHADE's adaptive schemes have dominated over many other adaptive schemes employed in DE. Likewise, the JADE mutation strategy with archive (see Eq. 35) has been adopted in many adaptive DE algorithms.

\subsubsection{Adaptive DE schema ranking}

Fig. 4 depicts an estimated rank of 24 adaptive DE algorithms based on their average performance in comparison with state-of-the-art DE algorithms (as presented in literature). In this figure, the sold arrow indicates that the algorithm in the source node has inherited the mutation strategy from the destination node; whereas, the intermittent line refers to the $\mathrm{DE}$ variants with less or almost equal performance in comparison with the DE algorithm in the source node. We can also observe that Fig 4 is divided into distinct layers. Each layer indicates that the $\mathrm{DE}$ algorithms placed in the same layer have almost equal performance.

Generally, Fig 4 shows that the adaptive DE algorithms with advanced mutation strategies such as JADE with archive (Zhang and Sanderson 2009b), MDE $p$ BX (Islam et al. 2012) and DEGL (Das et al. 2009b) have gained the overall best performance. This is because these advanced strategies could handle the deficiencies in the standard DE strategies such as the greediness in the DE/best/1 strategy and successfully managed to create the required balance between the exploration (i.e. the global search tendency) and exploitation (i.e. the local search tendency) capabilities of the algorithm. Therefore, we can observe from the same figure that the DE algorithms placed in the top layers such as SHADE (Tanabe and Fukunaga 2013) have already adopted these advanced strategies (e.g. DE/current-to-pBest/1 strategy in Eq. 35) and incorporate them with the parameter adaptive 
schemes to achieve the best algorithmic design performance. Likewise, adopting effective algorithms' schemes also applies to the parameter control schemes. LSHADE-cnEpSin (Awad et al. 2017b), L-covnSHADE (Awad et al. 2017c) and LSHADE-SPA (Mohamed et al. 2017) algorithms are already a modified versions of LSHADE algorithm' parameter control schemes (Tanabe and Fukunaga 2014). Another important type of adaptive DE variants is the dynamic selection of multiple DE strategies during the evolution process such as in ESPSDE (Mallipeddi et al. 2011), HSPEADE (Mallipeddi 2013) and CoDE (Wang et al. 2011). This type of adaptive algorithms has always show good performance and still a very hectic research trend; because, it has already been proved through experiments that at each stage of evolution there is a suitable DE strategy to impose different search step size and guide the search towards better direction. As such, EDEV algorithm (Wu et al. 2018) outperforms JADE with archive, CoDE, SaDE and EPSDE algorithms with outstanding performance as this algorithm proposed an adaptive scheme that manages to select the suitable adaptive DE scheme during the run. 


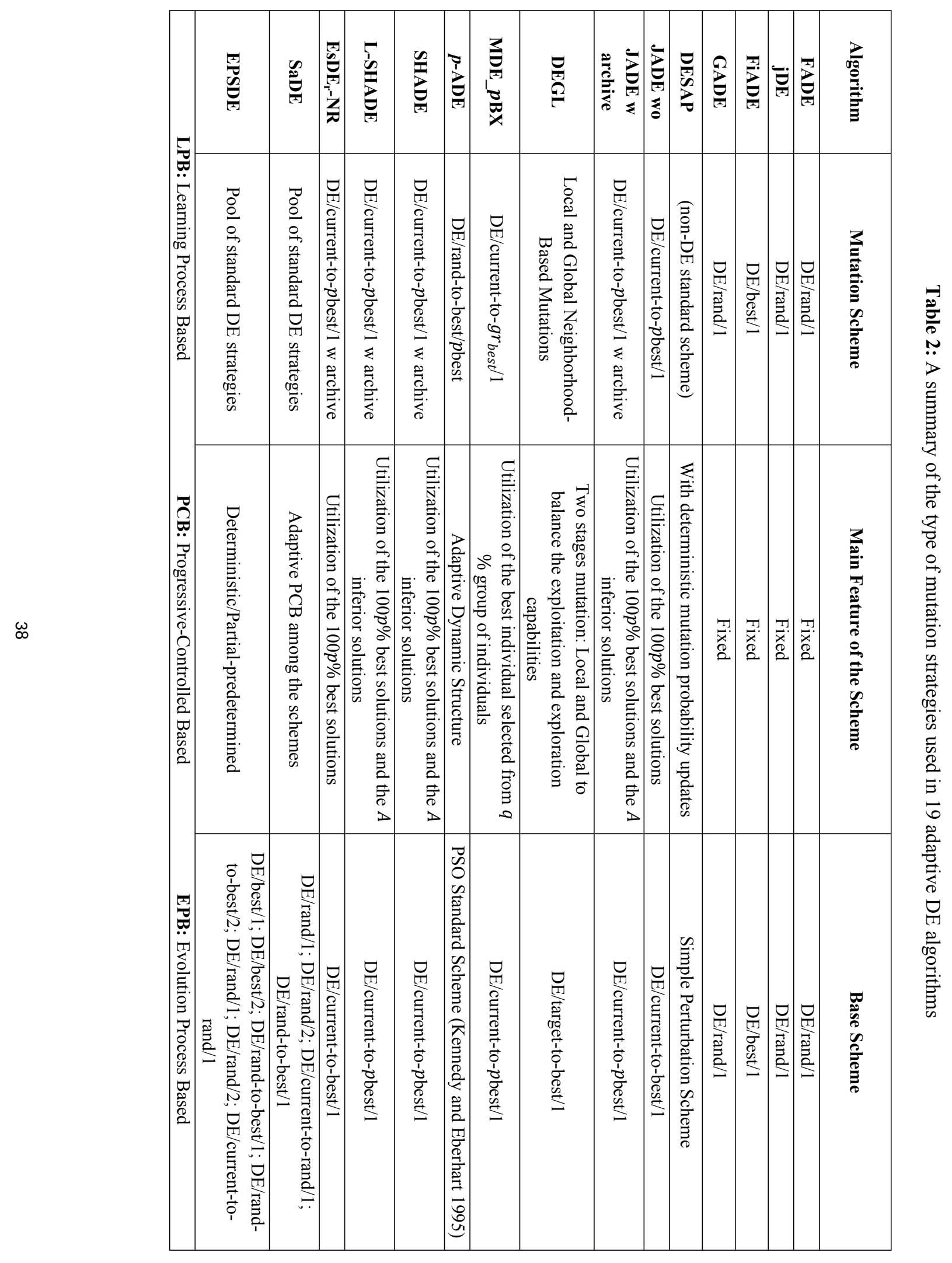




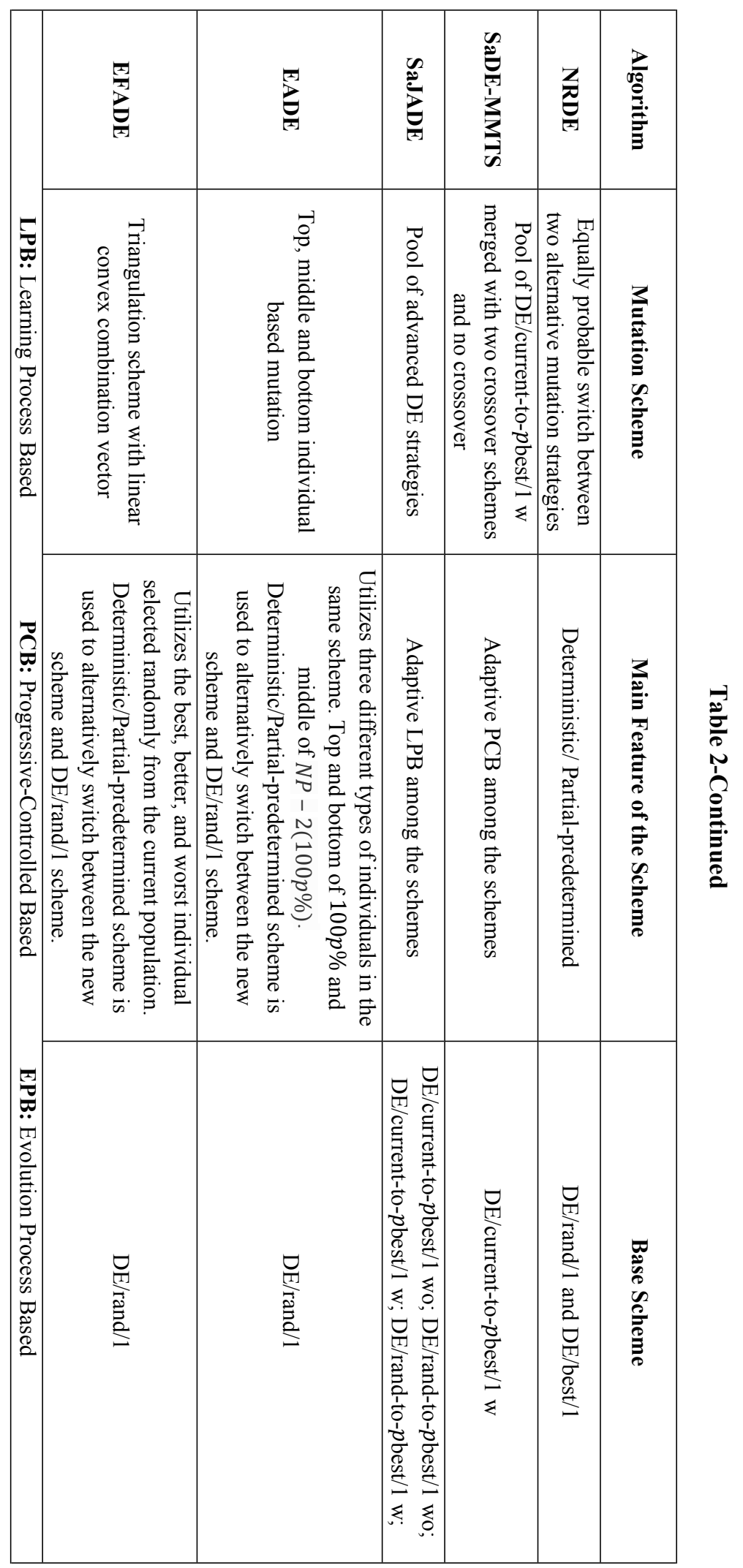




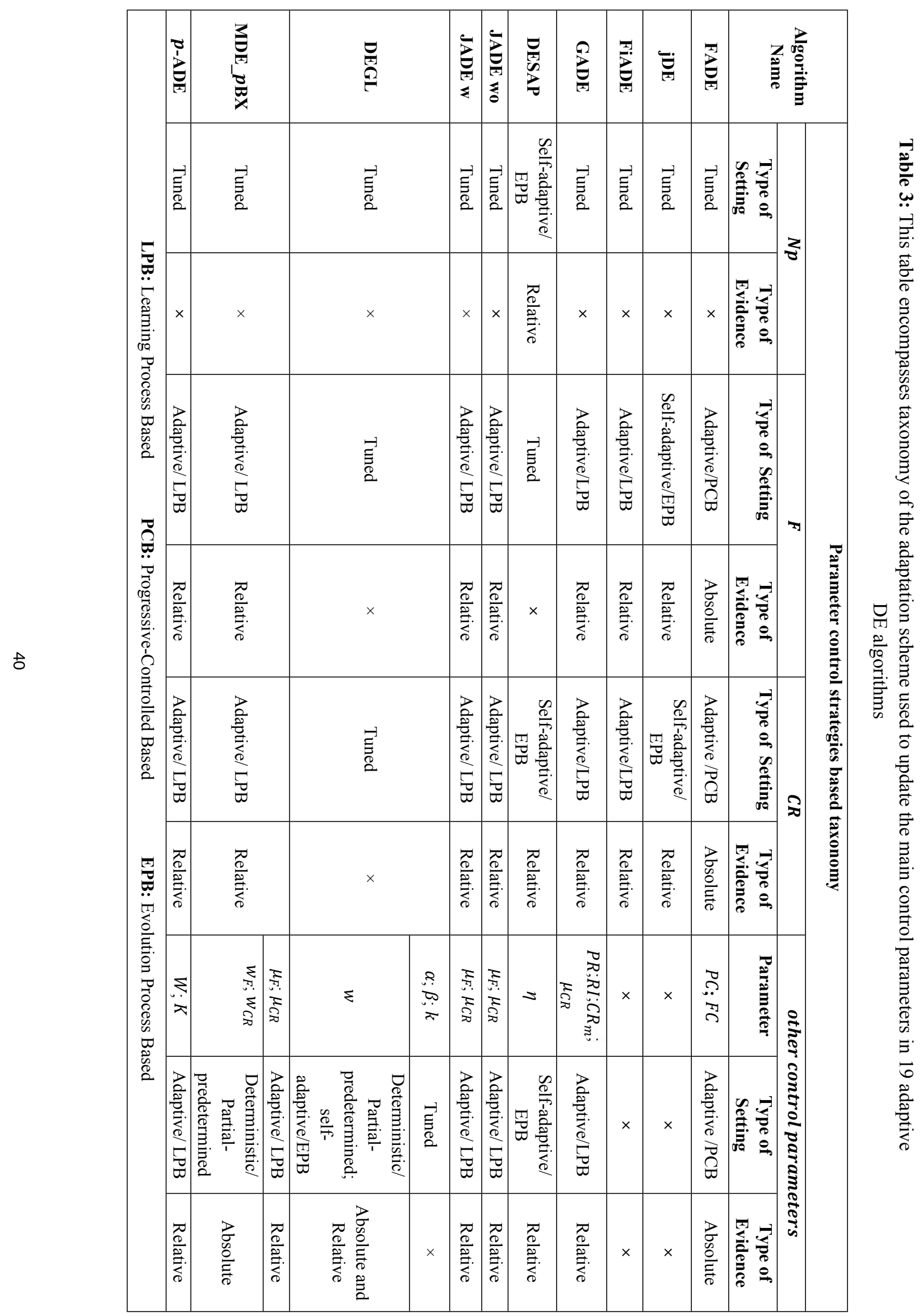




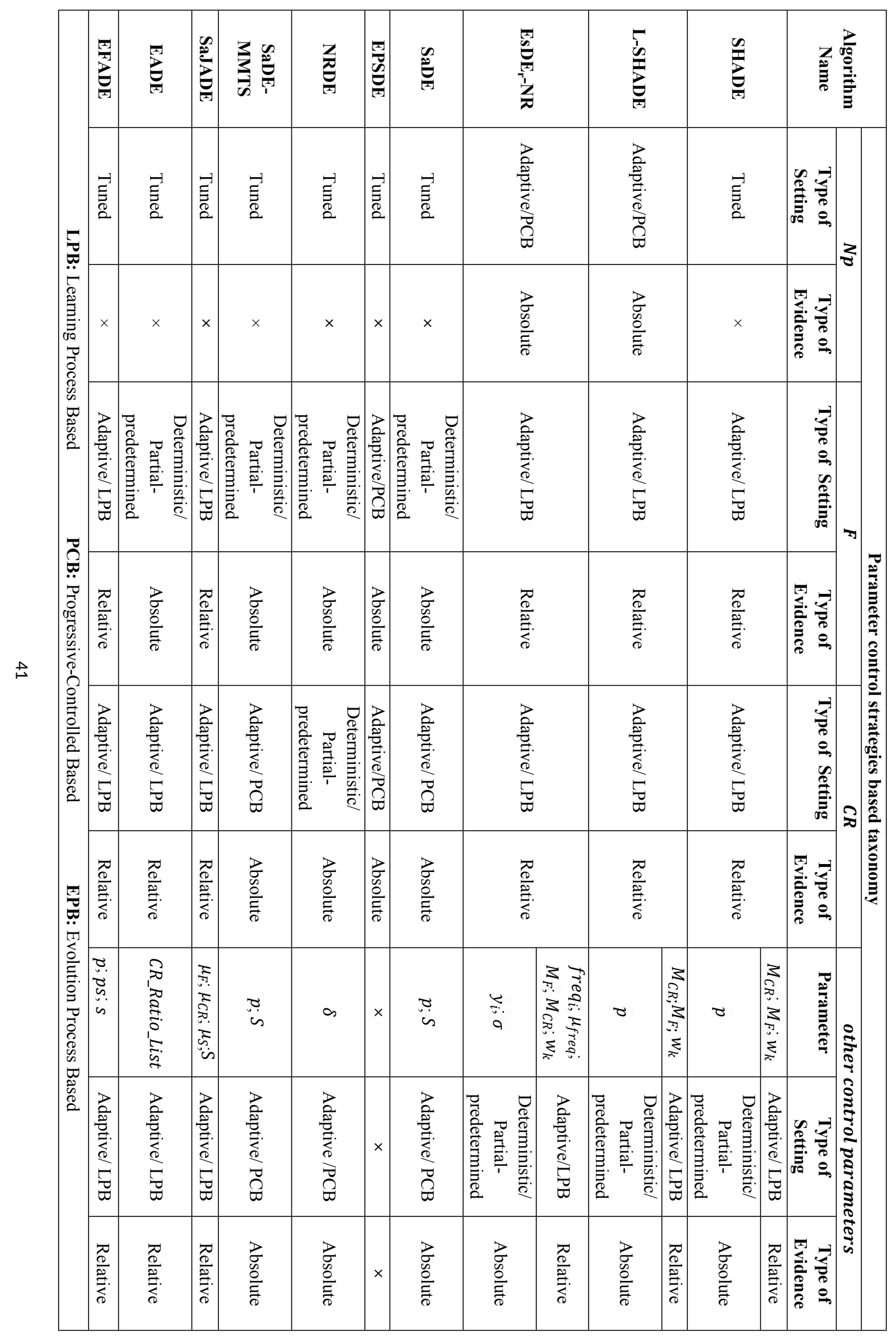




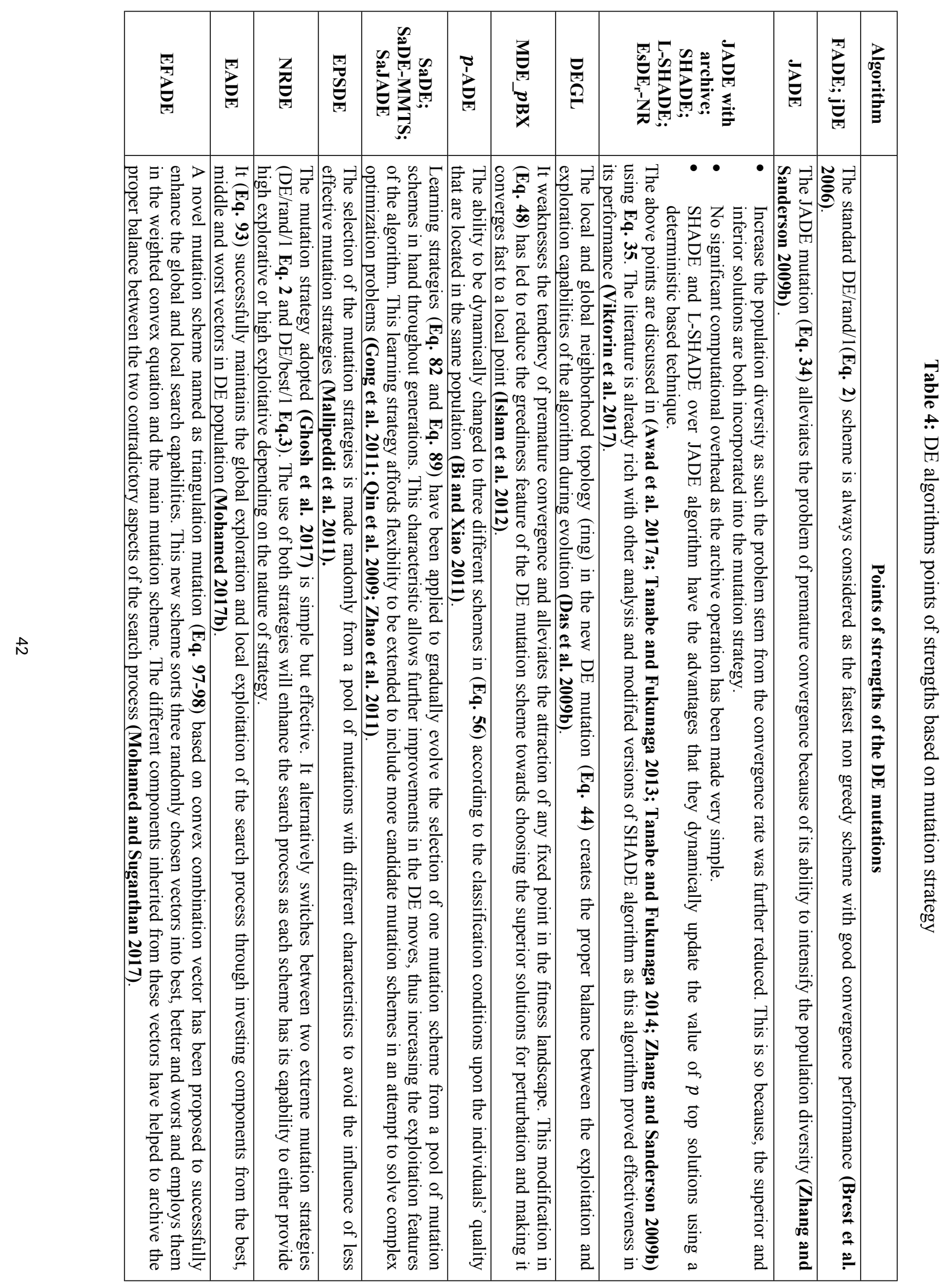




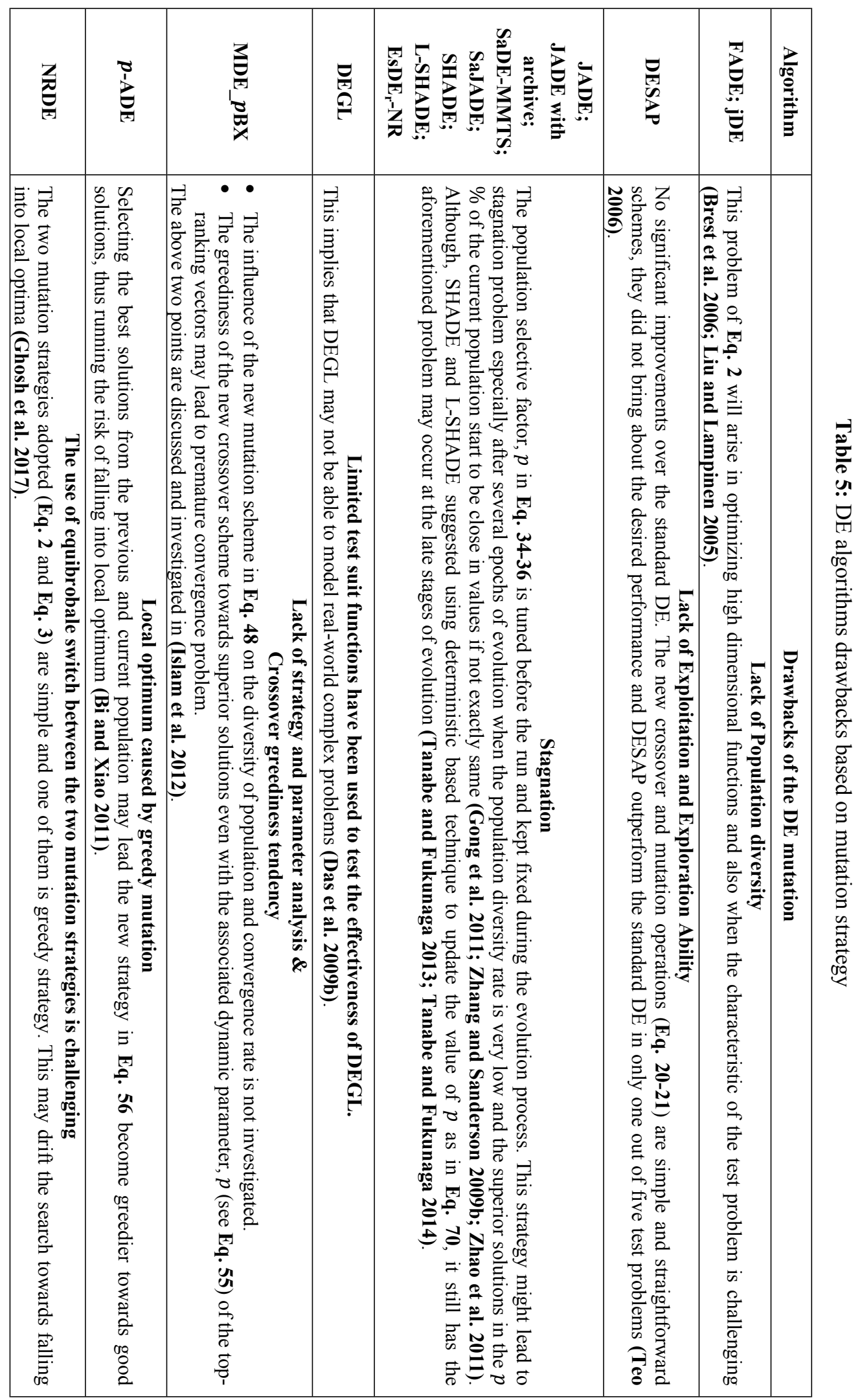




\begin{tabular}{|c|c|c|c|c|c|c|c|c|c|c|}
\hline 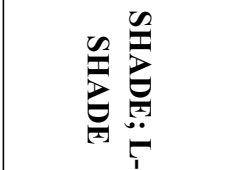 & $\mathbb{T}^{\pi}$ & $\frac{3}{2}$ & 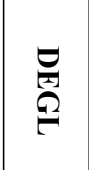 & 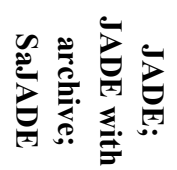 & 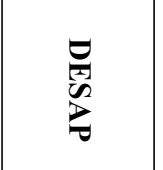 & $\sum_{\mathbb{T}}^{R}$ & $e^{T}$ & 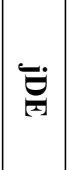 & $\vec{z}^{x}$ & \\
\hline 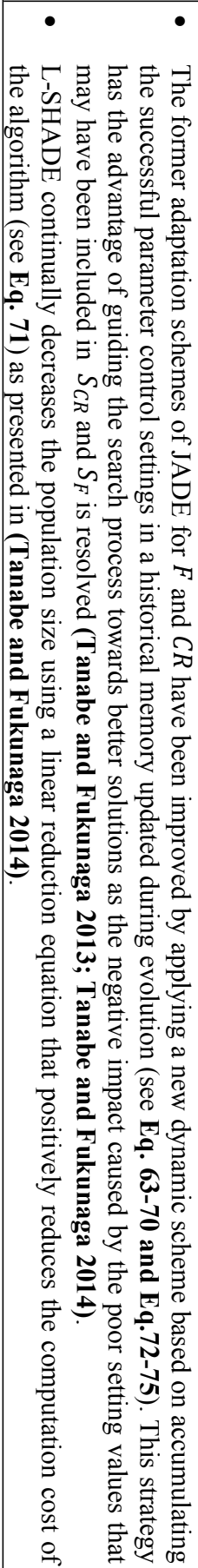 & 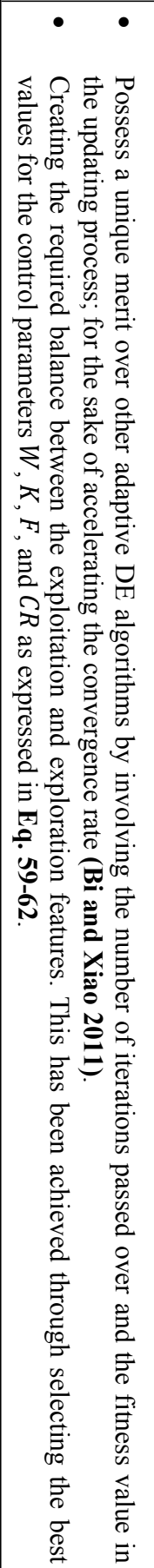 & 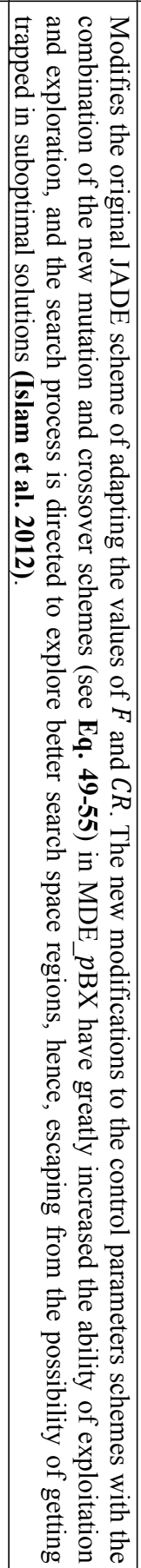 & 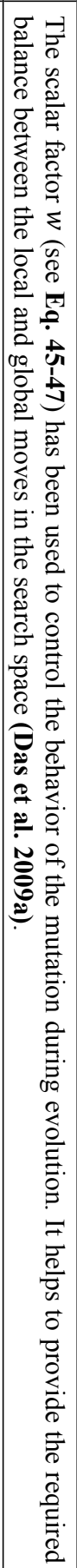 & 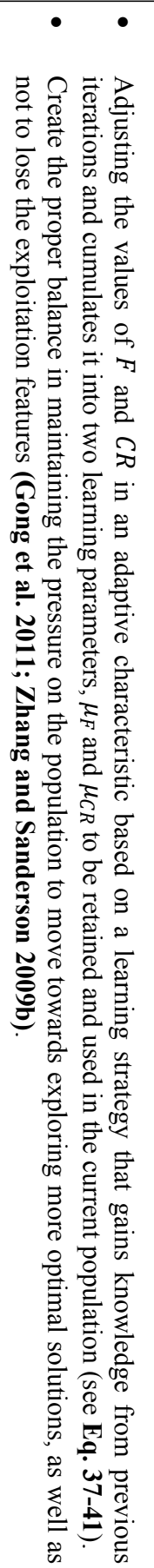 & 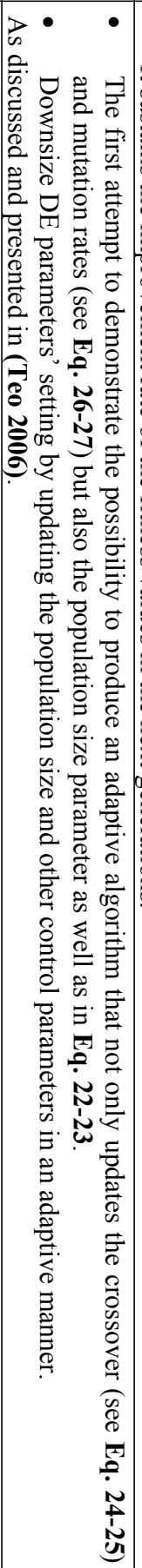 & 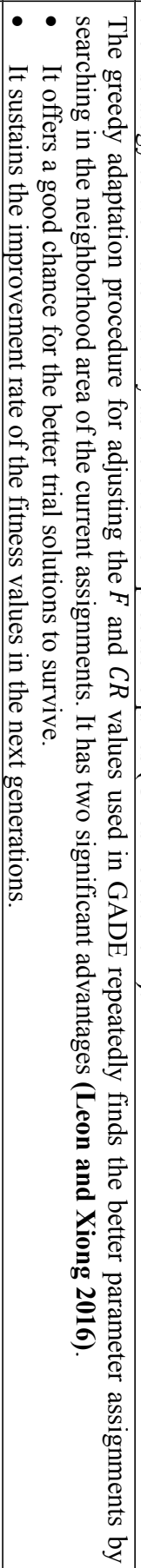 & 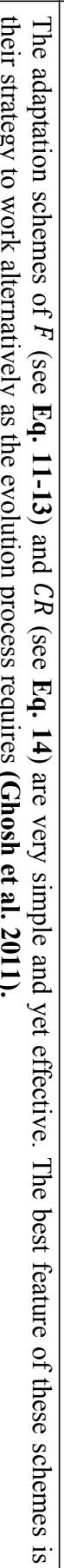 & 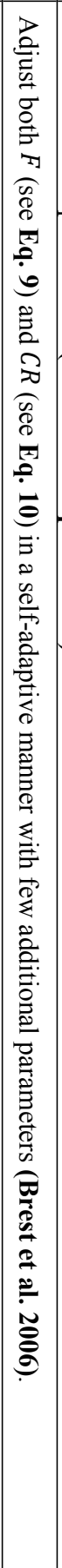 & 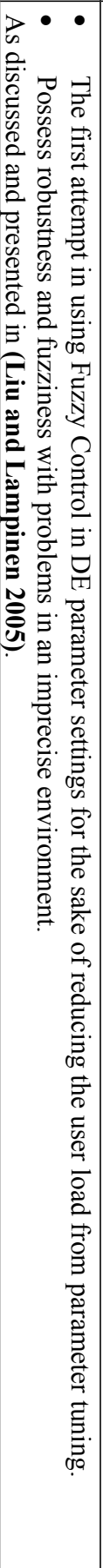 & 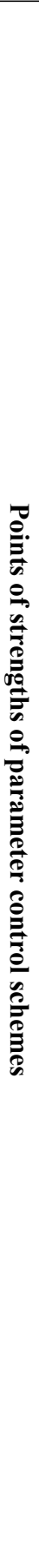 \\
\hline
\end{tabular}




\begin{tabular}{|c|c|c|c|c|c|c|c|c|c|}
\hline $\overrightarrow{0}_{\vec{x}}^{\mathrm{T}}$ & $\sum_{x}^{x}$ & 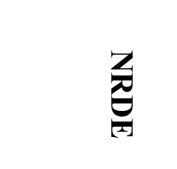 & & 氠 & 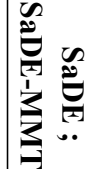 & & 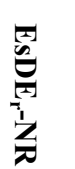 & & 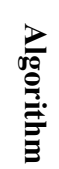 \\
\hline 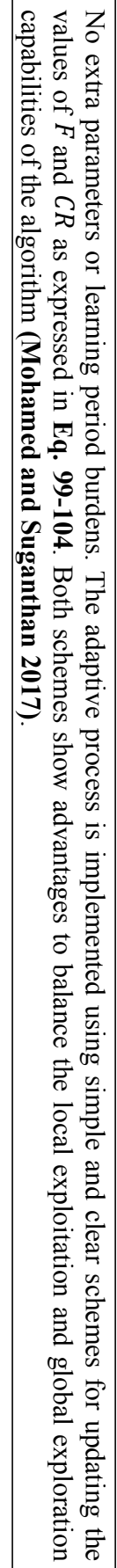 & 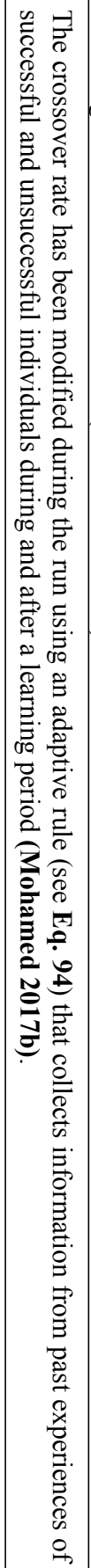 & 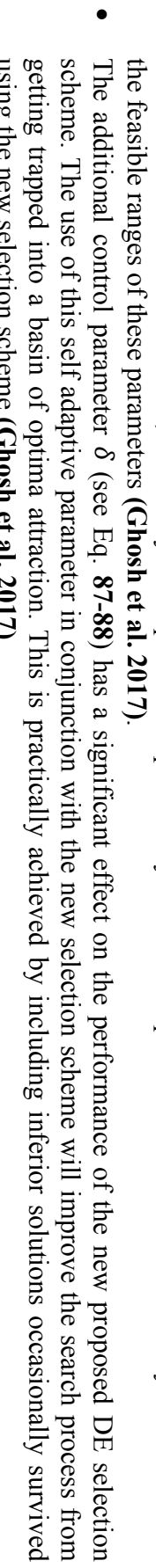 & 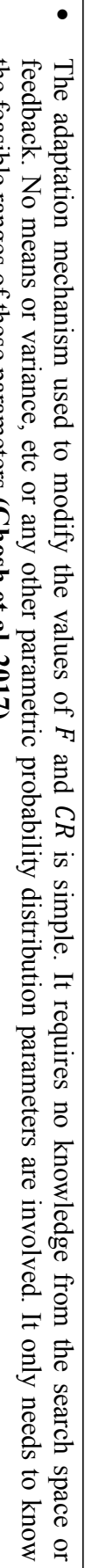 & 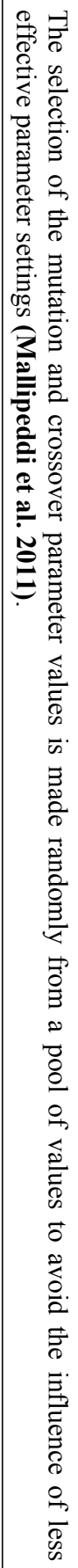 & 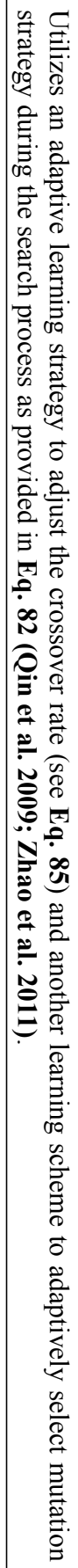 & 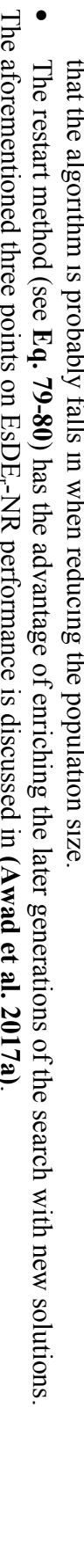 & 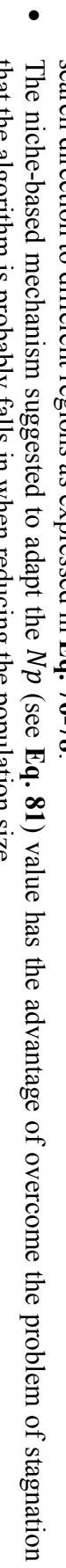 & 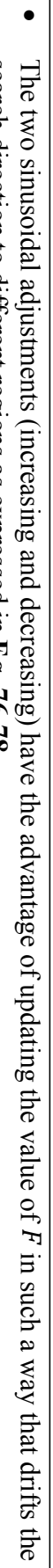 & 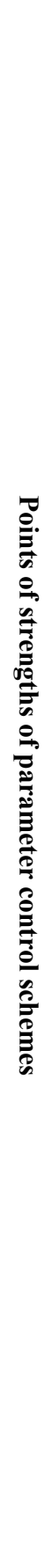 \\
\hline
\end{tabular}




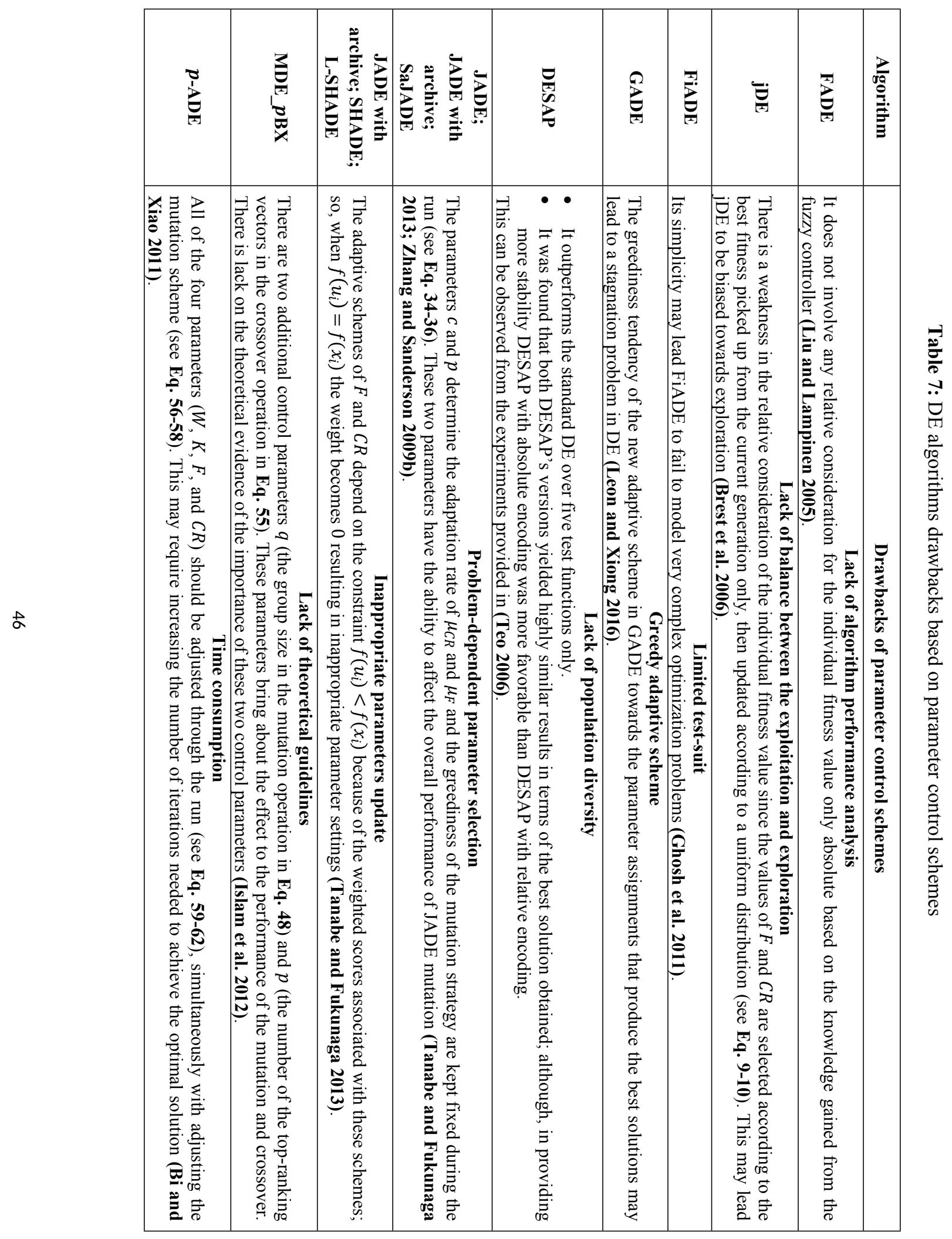




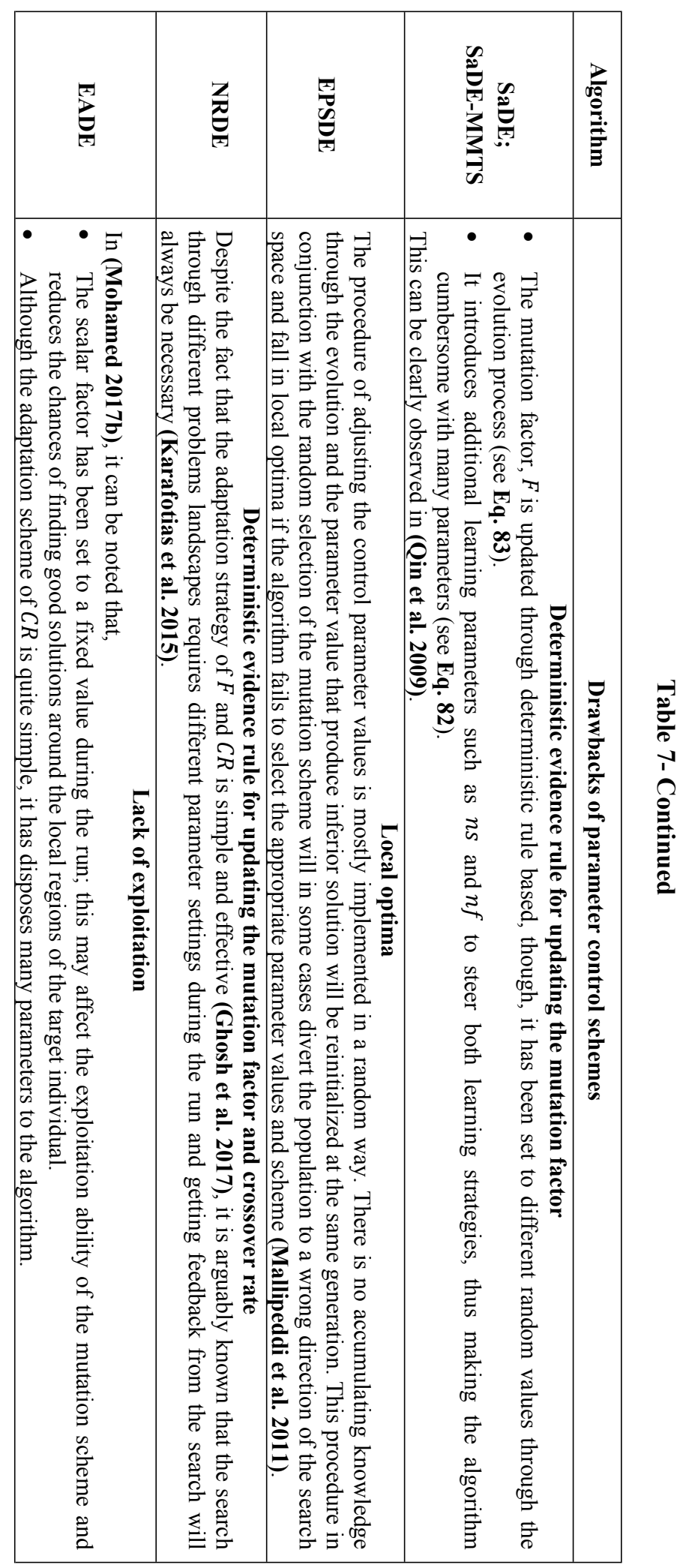




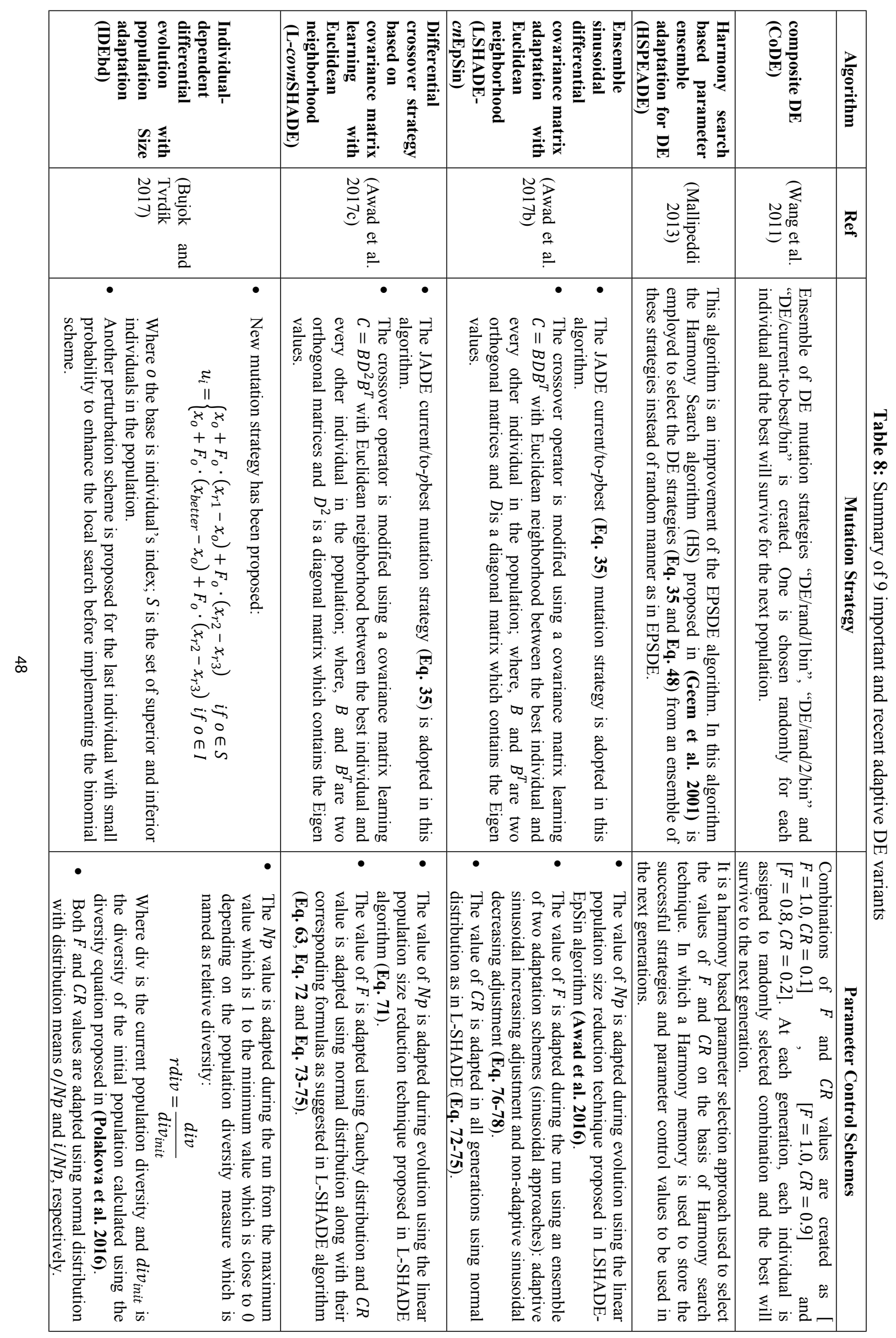




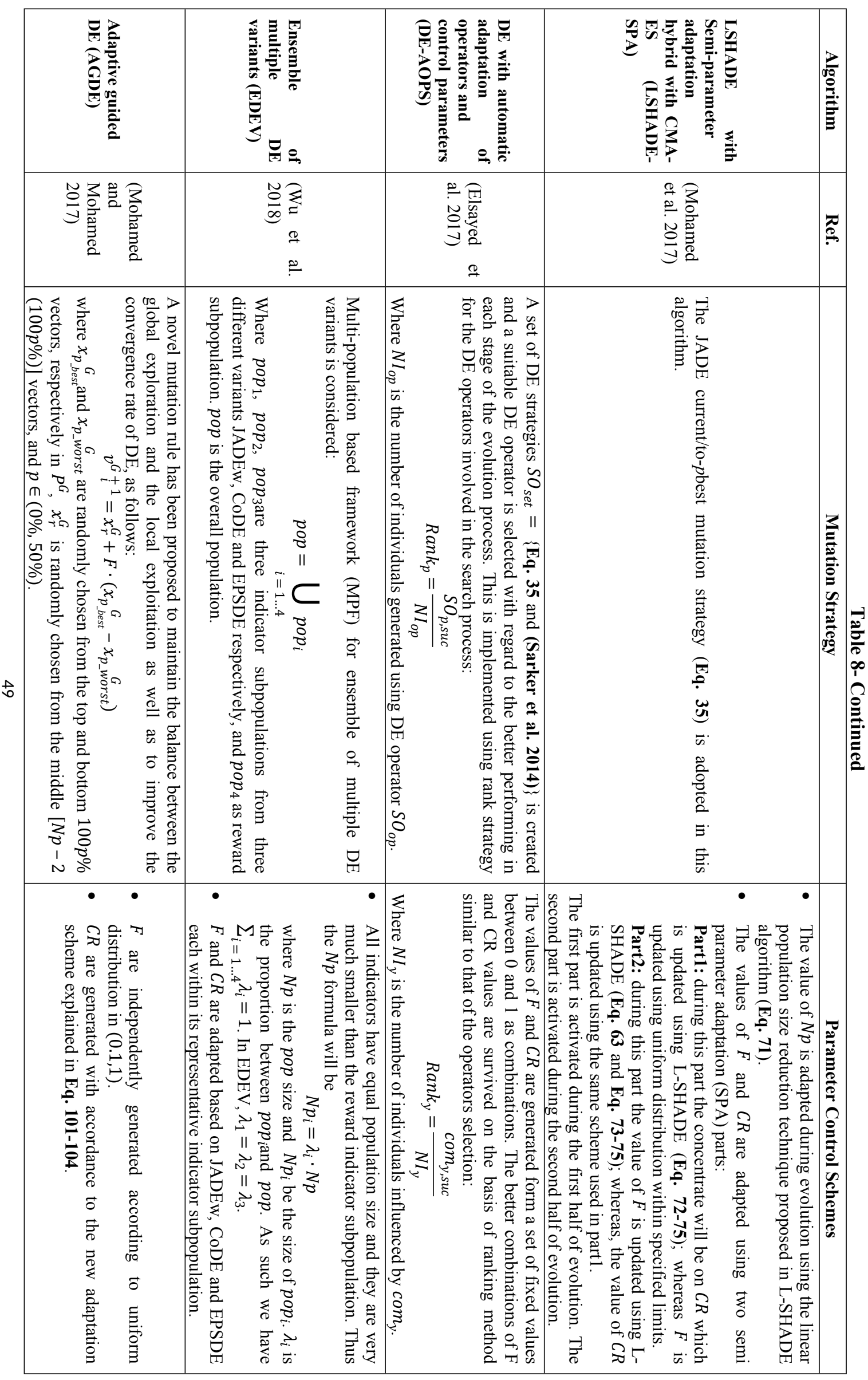




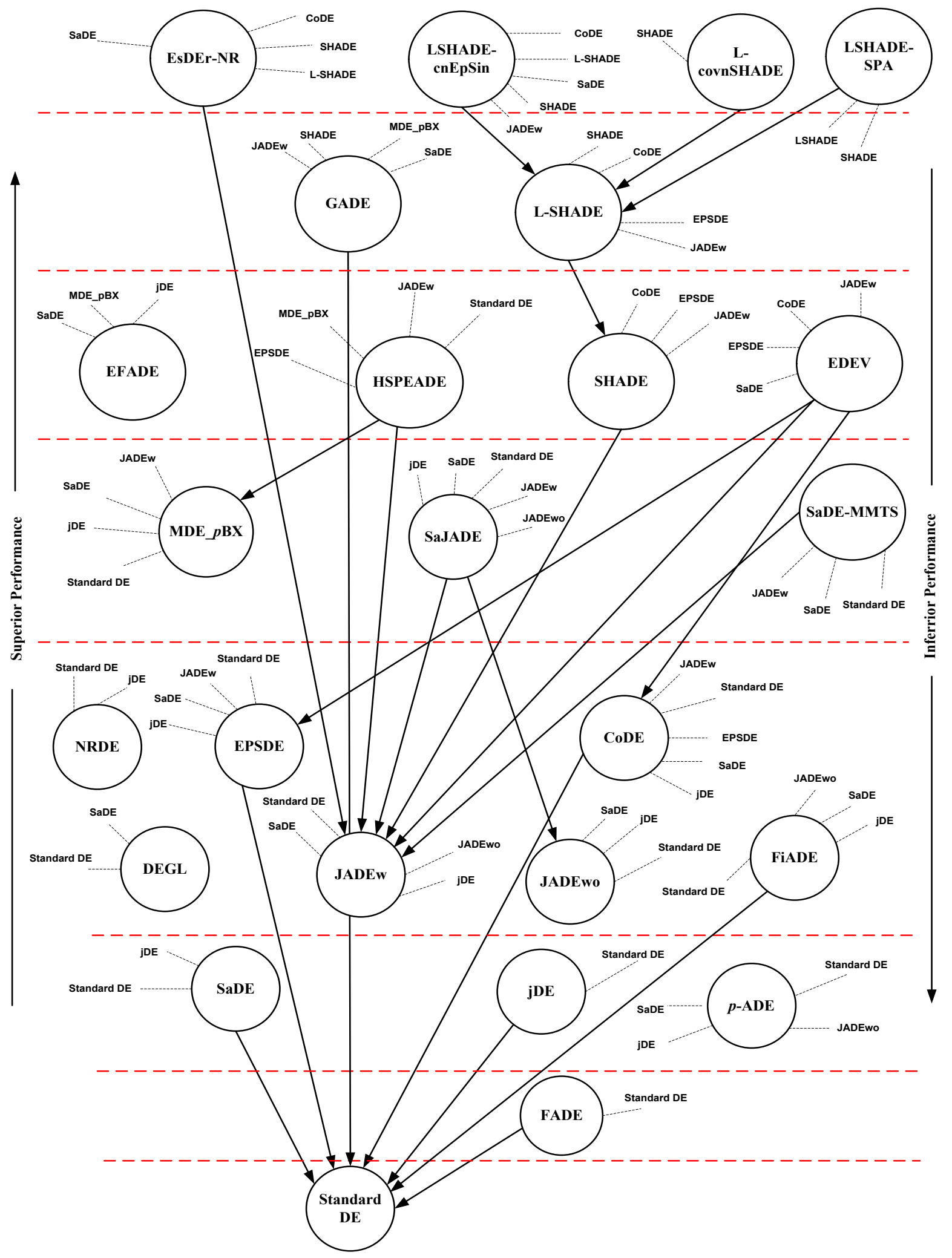

Fig 4: An estimated rank of the adaptive DE algorithms 


\title{
6 Metaheuristic framework with parameter control
}

After an interpretation of the literature and a comparative analysis of the aforementioned algorithms' design, we offer some guidelines on how to design and implement adaptive DE algorithms. The proposed designing framework provides the reader with the main steps that are required for integrating any proposed meta-algorithm with parameter and/or strategy adaptation schemes. These steps have been inspired by many adaptive algorithms already presented in literature. This constitutes also the various components of these algorithms, including selection, recombination, mutation, and survival operators.

Framework 1: General framework for constructing an adaptive metaheuristic with respect to parameters control and perturbation strategies

\begin{abstract}
Step 1: INDIVIDUAL (SOLUTION) ENCODING, POPULATION REPRESENTATION AND SELF-ADAPTIVE PARAMETERS

A population is a set (i.e. array) of individuals (chromosomes, particles) where $N p$, refers to the number of individuals in each generation. First, we have to encode the necessary information required for the problem analysis in the individual structure. Each individual should represent a complete solution to the problem at hand. All together, these parameters are called solution parameters (Eiben and Smith 2003). Additionally, if our intention is to work on self-adaptive algorithm, the individual should implicitly include also the encoding of the control parameter(s), or the so-called strategy's parameter(s) that we would to undergo evolution via recombination and mutation. This requires an intelligent decision to be taken into account in such a way that better parameter values would tend to produce better individuals (i.e. solutions) with the highest chance to survive and propagate for more offsprings (Brest et al. 2007; Brest et al. 2010).
\end{abstract}

\section{Step 2: INDIVIDUAL EVALUATION (SOLUTION VALIDATION) AND SELECTION}

The definition of the fitness function is crucially important for a successful application. In any meta-algorithm, we have to evaluate the fitness of each individual. There are many standard fitness functions that can be used to test any proposed meta-method (Cheng et al. 2017; Tanabe and Fukunaga 2013). Throughout many problems, it is more natural to state the fitness function as minimization with/without constraints rather than maximization of some utility objectives; however this depends on the type of the problem/application at hand. This step is always connected to selection operation and survival of the fittest, as these two operations work dependent on the fitness evaluation (Elsayed et al. 2017; Zamuda 2017).

\section{Step 3: INDIVIDUAL GENERATOR STRATEGY}

One of the most important steps when using metaheuristic algorithms is how the candidate solutions will be changed in order to diversify the population with new solutions. Exploration and exploitation are the two cornerstones of problem solving by search. For a successful evolutionary process, one has to get proper balance between exploration (to cover sufficiently the solution space seeking out for better solutions), and exploitation (refining the solutions by combining information gathered from good ones during the exploration phase). Also, diversity maintenance is important to prevent premature convergence. This gives a motivation to study and provide sufficient exploration and exploitation techniques to be incorporated into the meta-algorithm main paradigm (Mohamed 2017b; Mohamed and Suganthan 2017). The main individual generation strategies that should get a thorough understanding and then well applied are: recombination and mutation. Several individual strategies can be involved in the same algorithm. The selection of the right individual generator can be implemented implicitly during the run. This requires additional approaches that collect information during evolution in order to make decision which is the best strategy to be applied for an epoch (Ghosh et al. 2017; Wu et al. 2018).

\section{Step 4: STOPPING CRITERIA}

The most common stopping condition used in the literature is to allow the algorithm to run to a maximum number of iterations (Eiben and Smith 2003). A small number of iterations may not offer enough time for the algorithm to attain an optimum especially when the size of the search space is large. On the other hand, once the optimum solution is reached there will be no more gain from a very large number of iterations. In general, the maximum number of iterations allowed to 
multiply by the swarm size gives an indication of the number of particles evaluated by the algorithm (Brest and Maucec 2011).

\section{Step 5: INVESTIGATE THE INFLUENCE OF DIFFERENT PARAMETERS OF METAHEURISTICS}

This includes:

- Population size $N p$. To draw the effect of $N p$ on the population diversity and performance of the proposed metaalgorithm, also to see whether increasing the size of the population may prevent, or at least, reduce the chance of the algorithm to be trapped in local minima (Awad et al. 2016; Tanabe and Fukunaga 2014).

- Selection and perturbation operators (recombination and mutation) with their qualitative and quantitative parameter setting. A determination should be made in advance to the type of parameter control settings that would be changed in deterministic, adaptive and/or self-adaptive manner, the change evidence, and for which parameter(s) e.g. mutation factor, crossover rate, tournament size, and so on (Bujok and Tvrdík 2017; Wang et al. 2011) .

- Determine the scope of change which is either to be on the gene level, individual level, or the whole population level (Brest et al. 2007; Das et al. 2009b).

\section{Step 6: EXPERIMENTAL RESULTS}

This step is performed to reach twofold goals. First, to show the reliability and efficiency of the proposed methods, experimental results will be evaluated using various test bed problems (Cheng et al. 2017). Second, the relative performance of the proposed methods, when compared with other well-known state-of-the-art algorithms should be evaluated (both in qualitative and/or quantitative terms), and reported when applied to the same test problem/application. In all these experiments, the influences of different versions, components, and parameters of the meta-algorithm on performance are to be investigated, analyzed, and reported (Neri and Tirronen 2010; Zamuda and Brest 2015).

\section{Summary, conclusion and future insights}

The classification of adaptive metaheuristics and adaptive differential evolution (DE) algorithms is always an on-going research area. The research on developing adaptive meta-algorithms has been such a hot topic. As more and more new adaptive algorithms are proposed with new characteristics, the need for a general classification that can cover all these types of algorithms becomes a large demand. These classifications provide knowledge to those researches who are interested in this type of algorithms on what have been implemented and what improvements or developments can be added in this area as future work. In this study, two classifications have been proposed for the purpose: First, extension taxonomy to the EA parameter settings that covers in general the type of parameters settings in evolutionary computations (SECTION 3). Second, general classification to the adaptive DE algorithms that classifies these algorithms based on the parameters control of the algorithm as well as the number of DE strategies employed in the implementation (SECTION 5). Also, this study has implemented a number of tables (Tables 2-8) after a thorough algorithmic design investigation applied on 28 recent and important adaptive DE variants. These tables summarize everything related to the algorithmic design of these algorithms, and the points of weaknesses and strengths of each algorithm.

In summary, the main focus of our literature analysis is the algorithmic design and we attempt to offer a protocol for future DE implementations (SECTION 6). In addition, many studies can be conducted to extend or enhance the adaptive DE algorithms we have presented, some of these future directions are stated in Table 9. 


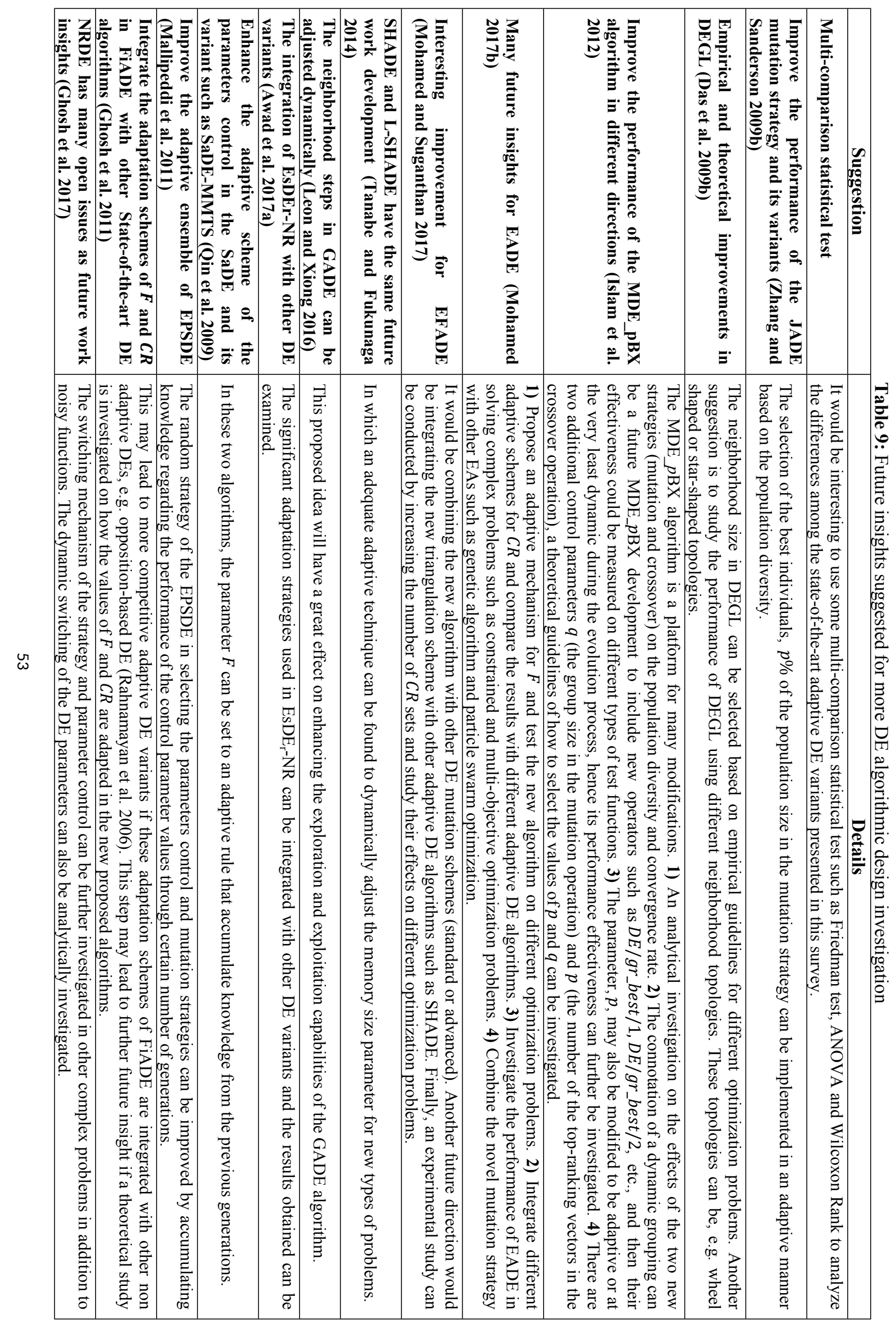

